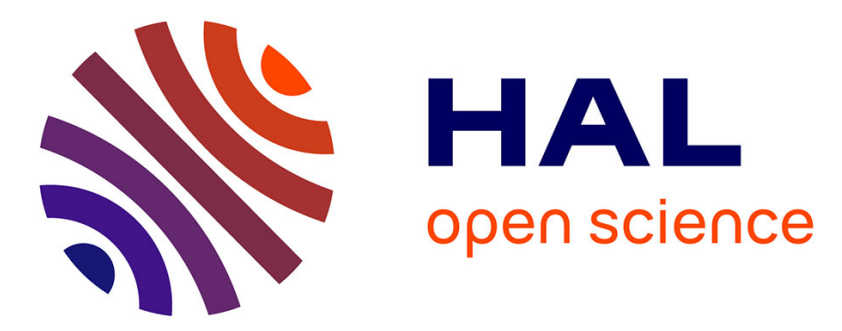

\title{
Extreme geometric quantiles in a multivariate regular variation framework
}

Stéphane Girard, Gilles Stupfler

\section{To cite this version:}

Stéphane Girard, Gilles Stupfler. Extreme geometric quantiles in a multivariate regular variation framework. Extremes, 2015, 18 (4), pp.629-663. 10.1007/s10687-015-0226-0 . hal-01155112v2

\section{HAL Id: hal-01155112 \\ https://hal.science/hal-01155112v2}

Submitted on 14 Sep 2015

HAL is a multi-disciplinary open access archive for the deposit and dissemination of scientific research documents, whether they are published or not. The documents may come from teaching and research institutions in France or abroad, or from public or private research centers.
L'archive ouverte pluridisciplinaire HAL, est destinée au dépôt et à la diffusion de documents scientifiques de niveau recherche, publiés ou non, émanant des établissements d'enseignement et de recherche français ou étrangers, des laboratoires publics ou privés. 


\title{
Extreme geometric quantiles in a multivariate regular variation framework
}

\author{
Stéphane Girard ${ }^{(1)} \&$ Gilles Stupfler ${ }^{(2)}$ \\ (1) Team Mistis, Inria Grenoble Rhône-Alpes \& LJK, Inovallée, 655, av. de l'Europe, \\ Montbonnot, 38334 Saint-Ismier cedex, France \\ (2) Aix Marseille Université, CNRS, EHESS, Centrale Marseille, GREQAM UMR 7316, \\ 13002 Marseille, France
}

\begin{abstract}
Considering extreme quantiles is a popular way to understand the tail of a distribution. While they have been extensively studied for univariate distributions, much less has been done for multivariate ones, primarily because there is no universally accepted definition of what a multivariate quantile or a multivariate distribution tail should be. In this paper, we focus on extreme geometric quantiles. In Girard and Stupfler (2015) Intriguing properties of extreme geometric quantiles, their asymptotics are established, both in direction and magnitude, under suitable integrability conditions, when the norm of the associated index vector tends to one. In this paper, we study extreme geometric quantiles when the integrability conditions are not fulfilled, in a framework of regular variation.
\end{abstract}

AMS Subject Classifications: 62H05, 62G32.

Keywords: Extreme quantile, geometric quantile, asymptotic behavior, multivariate regular variation.

\section{Introduction}

Let $X$ be a random vector in $\mathbb{R}^{d}$. Up to now, several definitions of multivariate quantiles of $X$ have been introduced in the statistical literature, see Serfling (2002) for a review of various possibilities for this notion. Here, we focus on the notion of "spatial" or "geometric" quantiles, introduced by Chaudhuri (1996), which generalizes the characterization of a univariate quantile shown in Koenker and Bassett (1978). For a given vector $v$ in the unit open ball $B^{d}$ of $\mathbb{R}^{d}$, where $d \geq 2$, a geometric quantile related to $v$ is a solution of the optimization problem defined by

$$
\underset{q \in \mathbb{R}^{d}}{\arg \min } \mathbb{E}(\|X-q\|-\|X\|)-\langle v, q\rangle,
$$


where $\langle\cdot, \cdot\rangle$ is the usual scalar product on $\mathbb{R}^{d}$ and $\|\cdot\|$ is the associated Euclidean norm. Any solution $q(v)$ of the problem (1) is called a $v$-th quantile. A $v$-th quantile $q(v) \in \mathbb{R}^{d}$ thus possesses both a direction and magnitude. It can be seen that geometric quantiles are actually special cases of $M$-quantiles introduced by Breckling and Chambers (1988) which were further analyzed by Koltchinskii (1997). Such quantiles have various appealing properties: for any $v \in B^{d}$, the $v$-th quantile is unique whenever the distribution of $X$ is not concentrated on a single straight line in $\mathbb{R}^{d}$ (see Chaudhuri, 1996), geometric quantiles are equivariant under any orthogonal transformation (Chaudhuri, 1996) and they characterize the associated distribution, namely, if two random vectors $X$ and $Y$ yield the same quantile function $q$, then they have the same distribution (Koltchinskii, 1997). Remark that on the one hand, for $v=0$, the well-known geometric median is obtained, which is the simplest example of a "central" quantile (see Small, 1990) and can be computed in an efficient way, see Cardot et al. (2013). On the other hand, for $v \neq 0$, the norm $\|v\|$ measures the deviation of the quantile $q(v)$ from the geometric median of the distribution. Since $v$ has a direction in addition to its magnitude, this immediately leads to a notion of directional outlyingness of a point (Chaouch and Goga, 2010). Figure 1 provides an illustration on a real data set from the Pima Indians Diabetes Database ${ }^{1}$ already considered by Cheng and De Gooijer (2007) and Chaouch and Goga (2010), among others. It appears that geometric iso-quantile curves tend to give a fair idea of the shape of the data cloud for moderate values of $\|v\|$.

These properties make geometric quantiles reasonable candidates when trying to define multivariate quantiles, which is why their estimation has been studied in several papers. We refer for instance to Chakraborty (2001) for the introduction of a transformation-retransformation procedure to obtain affine equivariant estimates of multivariate quantiles. It is stressed that the shape of the transformed quantile contours corresponds to that of the level sets of the probability distribution function when the underlying distribution is elliptically contoured, see also Cambanis et al. (1981). Chakraborty (2003) generalizes geometric quantiles to a multiresponse linear model while Dhar et al. (2013) defines a multivariate quantile-quantile plot using geometric quantiles. Conditional geometric quantiles can also be defined by substituting a conditional expectation to the expectation in (1): we refer to Cadre and Gannoun (2000) for the estimation of the conditional geometric median and to Cheng and de Gooijer (2007) for the estimation of an arbitrary conditional geometric quantile. The estimation of a conditional median when there is an infinite-dimensional covariate is considered in Chaouch and Laïb (2013).

Our focus in this paper is on extreme geometric quantiles, that is, when $\|v\| \rightarrow 1$. The estimation of univariate extreme quantiles, which requires the estimation of the so-called extreme value index, has been extensively studied, see for instance the monograph by de Haan and Ferreira (2006). In this case, there is also a growing interest about linking the random variable of interest to a covariate in order to analyze multidimensional datasets, see Gardes and Girard (2012), Daouia et al. (2013) or Stupfler (2013), among others. Only a few papers however consider multivariate extreme quantiles. Most of them rely on the

\footnotetext{
${ }^{1}$ ftp.ics.uci.edu/pub/machine-learning-databases/pima-indians-diabetes
} 
study of extreme level sets of the probability density function of $X$ when it is absolutely continuous with respect to the Lebesgue measure. See for instance Cai et al. (2011) for an application to the estimation of extreme risk regions for financial data and Einmahl et al. (2013) who focus on bivariate distributions with an application to insurance data. We also refer to Chernozhukov (2005) for extreme quantile estimation in a linear quantile regression model.

Girard and Stupfler (2015) obtained the asymptotics, in direction and magnitude, of the geometric quantile $q(\lambda u)$ in the limit $\lambda \uparrow 1$, for any $u$ on the unit hypersphere $S^{d-1}$ of $\mathbb{R}^{d}$. They proved that, if $\mathbb{E}\|X\|^{2}<\infty$, then the magnitude of this quantile always behaves asymptotically like $(1-\lambda)^{-1 / 2}$, and so does the magnitude of the difference between $u$ and the direction of $q(\lambda u)$. In particular, extreme geometric quantiles from a vector of independent uniform random variables and from a multivariate Gaussian random vector have asymptotically the same magnitude, although their probability density functions clearly do not have the same behavior at infinity. Compared to the univariate theory of extreme quantiles, this is somewhat surprising since one could expect the quantile to feature the asymptotic decay of the probability density function of $X$.

In this study, we provide an equivalent of the direction and magnitude of an extreme geometric quantile when the integrability condition is violated. To this end, it is assumed that $X$ has a probability density function $f$ satisfying an hypothesis of multivariate regular variation. As a corollary of our results, it appears that, in this context, the magnitude of an extreme geometric quantile depends on the index of multivariate regular variation of $f$. In other words, when $\mathbb{E}\|X\|^{2}=\infty$, the magnitude of an extreme geometric quantile does indeed feature the asymptotic behavior of the probability density function of $X$, similarly to the univariate case. Some statistical implications of this result are highlighted: in particular, it is shown how to derive Weissman type estimators (Weissman, 1978) for extreme geometric quantiles.

The main results of our paper are stated in Section 2 and some numerical illustrations are given in Section 3. Proofs are deferred to Section 4.

\section{Main results}

When $X$ has a probability density function $f$ on $\mathbb{R}^{d}, d \geq 2$, problem (1) can be rewritten as

$$
\underset{q \in \mathbb{R}^{d}}{\arg \min } \int_{\mathbb{R}^{d}}(\|x-q\|-\|x\|) f(x) d x-\langle v, q\rangle .
$$

Note that, as Chaudhuri (1996) points out, the integral above is always finite even though $\|X\|$ may not have a finite expectation. In this context, since the distribution of $X$ is not concentrated on a single straight line, there is a unique solution $q(v)$ of (1) for every $v \in B^{d}$ (see Chaudhuri, 1996 and Theorem 2.17 in Kemperman, 1987): the vector $q(v)$ is called the geometric quantile of $X$ associated with $v$. Besides, 
in this case, $q(v)$ is the unique solution of the equation

$$
v+\int_{\mathbb{R}^{d}} \frac{x-q}{\|x-q\|} f(x) d x=0
$$

with unknown $q$ (here, $t /\|t\|=0$ when $t=0)$. Moreover, Girard and Stupfler (2015, Proposition 1) proved that problem (1) has a solution if and only if $v \in B^{d}$. A direct consequence of this result is that, if $v \rightarrow u$ with $v \in B^{d}$ and $u \in S^{d-1}$, then

$$
\|q(v)\| \rightarrow \infty \text { and } \frac{q(v)}{\|q(v)\|} \rightarrow u
$$

see Theorem 1 in Girard and Stupfler (2015), where the convergence of vector functions is to be understood elementwise. Their main result (Theorem 2 in Girard and Stupfler, 2015) is reported here for the sake of self-containedness.

Proposition 1. Let $u \in S^{d-1}$ and let $\Pi_{u}: y \mapsto y-\langle y, u\rangle u$ denote the orthogonal projection onto the hyperplane of $\mathbb{R}^{d}$ having normal vector $u$.

(i) If $\mathbb{E}\|X\|<\infty$, then

$$
\|q(\lambda u)\|\left(\frac{q(\lambda u)}{\|q(\lambda u)\|}-u\right) \rightarrow \mathbb{E}\left(\Pi_{u}(X)\right) \text { as } \lambda \uparrow 1 .
$$

(ii) If $\mathbb{E}\|X\|^{2}<\infty$ and $M$ denotes the covariance matrix of $X$, then

$$
\|q(\lambda u)\|^{2}(1-\lambda) \rightarrow \frac{1}{2}\left(\operatorname{tr} M-u^{\prime} M u\right) \quad \text { as } \quad \lambda \uparrow 1
$$

where $u^{\prime}$ denotes the transpose of the vector $u$.

The goal of this paper is to obtain results analogue to those of Proposition 1 in the more general setting where the integrability assumptions are not fulfilled. To this end, we work in a framework of multivariate regular variation introduced in Cai et al. (2011). Surveys on multivariate regular variation include Jessen and Mikosch (2006) and the monograph by Resnick (2006). More precisely, the following condition is considered:

$\left(M_{\alpha}\right)$ The probability density function $f$ of $X$ is a continuous function on a neighborhood of infinity, such that the function $y \mapsto\|y\|^{d} f(y)$ is bounded in any compact neighborhood of 0 and there exist a positive function $Q$ on $\mathbb{R}^{d}$ and a function $V$ which is regularly varying at infinity with index $-\alpha<0$, such that

$$
\forall y \neq 0,\left|\frac{f(t y)}{t^{-d} V(t)}-Q(y)\right| \rightarrow 0 \text { and } \sup _{w \in S^{d-1}}\left|\frac{f(t w)}{t^{-d} V(t)}-Q(w)\right| \rightarrow 0 \text { as } t \rightarrow \infty
$$

Remark first that in condition $\left(M_{\alpha}\right)$, the function $V$ is determined only up to asymptotic equivalence. Since $V$ converges to 0 at infinity, we may and will assume in what follows that $V$ is bounded on $[0, \infty)$ and, by Theorem 1.3.3 p.14 in Bingham et al. (1987), continuous on a neighborhood of infinity. Moreover, if condition $\left(M_{\alpha}\right)$ holds, then $Q$ is a homogeneous continuous function of degree $-d-\alpha$ on $\mathbb{R}^{d} \backslash\{0\}$ and $f(y)=\|y\|^{-d} V(\|y\|) Q(y /\|y\|)(1+o(1))$ as $\|y\| \rightarrow \infty$, see Lemma 3 in Section 4. Since $V$ is regularly varying with index $-\alpha$, the function $f$ is then roughly of order $\|y\|^{-d-\alpha}$ as $\|y\| \rightarrow \infty$ and therefore the 
parameter $\alpha$ controls the asymptotic decay of $f$ at infinity. In particular, switching to polar coordinates, it is easily seen that $\mathbb{E}\|X\|^{\beta}$ is then finite if $\beta<\alpha$.

We are now in position to compute the asymptotic direction and magnitude of $q(\lambda u)$ as $\lambda \uparrow 1$ under the assumption $\left(M_{\alpha}\right)$. Let us highlight that, when $\alpha>1, \mathbb{E}\|X\|$ is finite and the asymptotic direction is provided by Proposition 1(i). Similarly, when $\alpha>2, \mathbb{E}\|X\|^{2}$ is finite and the asymptotic magnitude is provided by Proposition 1(ii).

Theorem 1. Let $u \in S^{d-1}$.

(i) If $\left(M_{\alpha}\right)$ holds with $\alpha \in(0,1)$, then

$$
\frac{1}{V(\|q(\lambda u)\|)}\left(\frac{q(\lambda u)}{\|q(\lambda u)\|}-u\right) \rightarrow \int_{\mathbb{R}^{d}} \frac{\Pi_{u}(y)}{\|y-u\|} Q(y) d y \quad \text { as } \quad \lambda \uparrow 1 .
$$

(ii) If $\left(M_{\alpha}\right)$ holds with $\alpha \in(0,2)$, then

$$
\frac{1-\lambda}{V(\|q(\lambda u)\|)} \rightarrow \int_{\mathbb{R}^{d}}\left(1+\frac{\langle y-u, u\rangle}{\|y-u\|}\right) Q(y) d y \quad \text { as } \quad \lambda \uparrow 1 .
$$

Since $V$ is regularly varying with index $-\alpha$, it follows from Theorem 1(ii) that, in model $\left(M_{\alpha}\right)$, when $\alpha \in(0,2)$, the norm $\|q(\lambda u)\|$ of the extreme quantile behaves roughly like $(1-\lambda)^{-1 / \alpha}$ as $\lambda \uparrow 1$. In this case, we thus see that the magnitude of an extreme geometric quantile does indeed feature the behavior of $X$ far from the origin.

However, this result excludes the limit cases $\alpha=1$ for the asymptotic direction and $\alpha=2$ for the asymptotic magnitude. The method we use to handle these cases is somewhat different; in particular, we shall work with the functions $L: t \mapsto t^{\alpha} V(t)$ and

$$
\mathcal{L}: t \mapsto \int_{1}^{t} L(r) \frac{d r}{r}
$$

(this notation will be retained throughout the paper). Since $L$ is slowly varying as infinity, so is $\mathcal{L}$ and $\mathcal{L}(r) / L(r) \rightarrow \infty$ as $r \rightarrow \infty$, see Proposition 1.5.9a p.26 in Bingham et al. (1987). Furthermore, we define, if $\Sigma$ is a positive definite $d \times d$ symmetric matrix, the ellipsoid $E_{\Sigma}^{d-1}=\left\{x \in \mathbb{R}^{d} \mid x^{\prime} \Sigma^{-1} x=1\right\}$ and its related surface measure $\mu_{\Sigma}$ given by $\mu_{\Sigma}(C)=(\operatorname{det} \Sigma)^{1 / 2} \sigma\left(\Sigma^{-1 / 2} C\right)$ for every Borel measurable subset $C$ of $E_{\Sigma}^{d-1}$, where $\sigma$ is the standard surface measure on $S^{d-1}$. Then, for every integrable function $h$ on $\mathbb{R}^{d}$ and every $a<b \in[0, \infty]$, we have

$$
\int_{\mathbb{R}^{d}} h(x) \mathbb{1}_{[a, b]}\left(\left(x^{\prime} \Sigma^{-1} x\right)^{1 / 2}\right) d x=\int_{a}^{b} \int_{E_{\Sigma}^{d-1}} h(r w) r^{d-1} d r \mu_{\Sigma}(d w) .
$$

Our second main result is the following:

Theorem 2. Let $u \in S^{d-1}$ and $\Sigma$ be an arbitrary positive definite $d \times d$ symmetric matrix.

(i) If $\left(M_{1}\right)$ holds and $\mathcal{L}(t) \rightarrow \infty$ as $t \rightarrow \infty$ then

$$
\frac{\|q(\lambda u)\|}{\mathcal{L}(\|q(\lambda u)\|)}\left(\frac{q(\lambda u)}{\|q(\lambda u)\|}-u\right) \rightarrow \int_{E_{\Sigma}^{d-1}} \Pi_{u}(w) Q(w) \mu_{\Sigma}(d w) \quad \text { as } \quad \lambda \uparrow 1 .
$$


(ii) If $\left(M_{2}\right)$ holds and $\mathcal{L}(t) \rightarrow \infty$ as $t \rightarrow \infty$ then

$$
\frac{\|q(\lambda u)\|^{2}}{\mathcal{L}(\|q(\lambda u)\|)}(1-\lambda) \rightarrow \frac{1}{2} \int_{E_{\Sigma}^{d-1}}\left\langle\Pi_{u}(w), w\right\rangle Q(w) \mu_{\Sigma}(d w) \quad \text { as } \lambda \uparrow 1 .
$$

Let us remark that both limits are independent of the arbitrary matrix $\Sigma$. A convenient choice is to take $\Sigma$ as the identity matrix and thus to integrate on the unit sphere, but it may be interesting to consider other, nontrivial cases, see the discussion about elliptically contoured distributions in Corollary 2.

It may be seen from this result that, in the particular class $\left(M_{2}\right)$ and when $\mathcal{L}(t) \rightarrow \infty$ as $t \rightarrow \infty$ (which ensures that $\mathbb{E}\|X\|^{2}=\infty$ ), the magnitude of an extreme geometric quantile does again feature the behavior of $X$ far from the origin, through the function $L$. The influence of $L$ is illustrated in the following example:

Example If $L(t)=c_{\beta}(\log t)^{\beta} \mathbb{1}_{(1, \infty)}(t)$, where $\beta>-1$ and $c_{\beta}>0$, then

$$
\|q(\lambda u)\|^{2}(\log \|q(\lambda u)\|)^{-\beta-1}(1-\lambda) \rightarrow c_{\beta}^{\prime} \int_{S^{d-1}}\left\langle\Pi_{u}(w), w\right\rangle Q(w) \sigma(d w) \text { as } \lambda \uparrow 1
$$

where $c_{\beta}^{\prime}>0$. Lemma 2 (see Section 4) entails

$$
\|q(\lambda u)\|=C_{\beta}(u)(1-\lambda)^{-1 / 2}\left[\log \left(\frac{1}{1-\lambda}\right)\right]^{(\beta+1) / 2}(1+\mathrm{o}(1)) \text { as } \lambda \uparrow 1
$$

where $C_{\beta}(u)>0$. Consequently, in this case, the larger is $\beta$ (and thus, the slower $f$ converges to 0 at infinity), the larger is the order of the extreme geometric quantile.

Collecting the results from Proposition 1, Theorem 1 and Theorem 2, we obtain the following, somehow unified result:

Corollary 1. Let $u \in S^{d-1}$.

(i) If $\left(M_{\alpha}\right)$ holds with $\alpha \in(0,1)$, then

$$
\begin{aligned}
\frac{1}{V(\|q(\lambda u)\|)}\left(\frac{q(\lambda u)}{\|q(\lambda u)\|}-u\right) & \rightarrow \int_{\mathbb{R}^{d}} \frac{\Pi_{u}(y)}{\|y-u\|} Q(y) d y \\
\text { and } \frac{1-\lambda}{V(\|q(\lambda u)\|)} & \rightarrow \int_{\mathbb{R}^{d}}\left(1+\frac{\langle y-u, u\rangle}{\|y-u\|}\right) Q(y) d y \quad \text { as } \quad \lambda \uparrow 1 .
\end{aligned}
$$

(ii) If $\left(M_{1}\right)$ holds and $\mathcal{L}(t) \rightarrow \infty$ as $t \rightarrow \infty$, then for any arbitrary positive definite $d \times d$ symmetric matrix $\Sigma$,

$$
\begin{aligned}
\frac{\|q(\lambda u)\|}{\mathcal{L}(\|q(\lambda u)\|)}\left(\frac{q(\lambda u)}{\|q(\lambda u)\|}-u\right) & \rightarrow \int_{E_{\Sigma}^{d-1}} \Pi_{u}(w) Q(w) \mu_{\Sigma}(d w) \\
\text { and } \frac{1-\lambda}{V(\|q(\lambda u)\|)} & \rightarrow \int_{\mathbb{R}^{d}}\left(1+\frac{\langle y-u, u\rangle}{\|y-u\|}\right) Q(y) d y \text { as } \lambda \uparrow 1 .
\end{aligned}
$$

(iii) If $\left(M_{\alpha}\right)$ holds with $\alpha \in(1,2)$, then

$$
\begin{aligned}
\|q(\lambda u)\|\left(\frac{q(\lambda u)}{\|q(\lambda u)\|}-u\right) & \rightarrow \int_{\mathbb{R}^{d}} \Pi_{u}(y) f(y) d y \\
\text { and } \frac{1-\lambda}{V(\|q(\lambda u)\|)} & \rightarrow \int_{\mathbb{R}^{d}}\left(1+\frac{\langle y-u, u\rangle}{\|y-u\|}\right) Q(y) d y \quad \text { as } \quad \lambda \uparrow 1 .
\end{aligned}
$$


(iv) If $\left(M_{2}\right)$ holds and $\mathcal{L}(t) \rightarrow \infty$ as $t \rightarrow \infty$, then for any arbitrary positive definite $d \times d$ symmetric matrix $\Sigma$,

$$
\begin{array}{ll}
\|q(\lambda u)\|\left(\frac{q(\lambda u)}{\|q(\lambda u)\|}-u\right) & \rightarrow \int_{\mathbb{R}^{d}} \Pi_{u}(y) f(y) d y \\
\text { and } \frac{\|q(\lambda u)\|^{2}}{\mathcal{L}(\|q(\lambda u)\|)}(1-\lambda) & \rightarrow \frac{1}{2} \int_{E_{\Sigma}^{d-1}}\left\langle\Pi_{u}(w), w\right\rangle Q(w) \mu_{\Sigma}(d w) \text { as } \lambda \uparrow 1 .
\end{array}
$$

It therefore appears that the norm of an extreme quantile can be asymptotically expanded as

$$
\|q(\lambda u)\|=\left[A_{2, \alpha}(u)\right]^{1 / \min (2, \alpha)} B_{2, \alpha}\left((1-\lambda)^{-1}\right) \text { when } u \in S^{d-1} \text { and } \lambda \uparrow 1,
$$

for all $\alpha>0$, where depending on whether $\alpha<2, \alpha=2$ or $\alpha>2, A_{2, \alpha}(u)$ is the limiting term in Proposition 1(ii), Theorem 1(ii) or Theorem 2(ii), and $B_{2, \alpha}$ is a regularly varying function with index $1 / \min (2, \alpha)$. The asymptotic direction of an extreme quantile can then be expanded as

$$
\frac{q(\lambda u)}{\|q(\lambda u)\|}=u+A_{1, \alpha}(u)\left[A_{2, \alpha}(u)\right]^{-\min (1, \alpha) / \min (2, \alpha)} B_{1, \alpha}\left((1-\lambda)^{-1}\right) \text { when } u \in S^{d-1} \text { and } \lambda \uparrow 1,
$$

for all $\alpha>0$, where depending on whether $\alpha<1, \alpha=1$ or $\alpha>1, A_{1, \alpha}(u)$ is the limiting term in Proposition 1(i), Theorem 1(i) or Theorem 2(i), and $B_{1, \alpha}$ is a regularly varying function with index $-\min (1, \alpha) / \min (2, \alpha)$. These expansions emphasize again the particular role of the values $\alpha=1$ and $\alpha=2$.

Besides, it is possible to link the expressions of $A_{1, \alpha}(u)$ obtained in the situations $\alpha>1$ and $\alpha=1$. To this end, we introduce a particular subclass of $\left(M_{\alpha}\right)$ :

$\left(M_{\alpha}^{\prime}\right)$ For all $x \neq 0, f(x)=Q(x) L\left(\left(x^{\prime} \Sigma^{-1} x\right)^{1 / 2}\right)$ where $\Sigma$ is a positive definite $d \times d$ symmetric matrix, $Q$ is a homogeneous continuous function of degree $-d-\alpha$ on $\mathbb{R}^{d} \backslash\{0\}$ and $L$ is a slowly varying function at infinity which is continuous in a neighborhood of infinity and is such that

$$
t \mapsto t^{-\alpha} L(t) \text { is bounded, } \int_{0}^{\infty} L(r) \frac{d r}{r^{1+\alpha}}<\infty \text { and } \int_{1}^{t} L(r) \frac{d r}{r} \rightarrow \infty \text { as } t \rightarrow \infty .
$$

Noting that, for every positive definite $d \times d$ symmetric matrix $\Sigma$, the continuous map $w \mapsto w^{\prime} \Sigma^{-1} w$ is positive and bounded on $S^{d-1}$, the uniform convergence theorem for slowly varying functions (see e.g. Theorem 1.5.2 p.22 in Bingham et al., 1987) entails that the class of probability density functions $\left(M_{\alpha}^{\prime}\right)$ satisfies the hypotheses of the original model $\left(M_{\alpha}\right)$, with $V(t)=t^{-\alpha} L(t)$. Using (5), it is straightforward that in this particular model, the hypothesis on $L$ entails that $\mathbb{E}\|X\|^{\beta}$ is finite if and only if $\beta<\alpha$.

Remark now that, in the class $\left(M_{\alpha}^{\prime}\right), \alpha>1$, equation (5) entails:

$$
\mathbb{E}\left(\Pi_{u}(X)\right)=\int_{0}^{\infty} L(r) \frac{d r}{r^{\alpha}} \int_{E_{\Sigma}^{d-1}} \Pi_{u}(w) Q(w) \mu_{\Sigma}(d w) .
$$

Thus, the limiting terms $A_{1, \alpha}(u)$ of Proposition 1(i) and Theorem 2(i) are similar. To link the expressions of $A_{2, \alpha}(u)$ obtained in the situations $\alpha>2$ and $\alpha=2$, we work in the subclass of elliptically contoured distributions: 
Corollary 2. Let $u \in S^{d-1}$. If $\left(M_{2}^{\prime}\right)$ holds and $Q$ is constant equal to 1 on the ellipsoid $E_{\Sigma}^{d-1}$, then

$$
\frac{\|q(\lambda u)\|^{2}}{\mathcal{L}(\|q(\lambda u)\|)}(1-\lambda) \rightarrow \frac{a_{d}}{2 d}(\operatorname{det} \Sigma)^{1 / 2}\left(\operatorname{tr} \Sigma-u^{\prime} \Sigma u\right) \quad \text { as } \lambda \uparrow 1
$$

where $a_{d}:=2 \pi^{d / 2} / \Gamma(d / 2)$ is the surface area of $S^{d-1}$ and $\Gamma$ is Euler's Gamma function.

This result shows that, when the distribution is elliptically contoured, the limiting terms $A_{2, \alpha}(u)$ of Proposition 1(ii) and Theorem 2(ii) are similar. In particular, the matrices $\Sigma$ and $M$ play the same role: it is indeed well-known that these matrices are closely related when the covariance matrix $M$ is finite, by the identity

$$
M=\left(\int_{0}^{\infty} L(r) \frac{d r}{r^{\alpha-1}}\right)(\operatorname{det} \Sigma)^{1 / 2} \frac{a_{d}}{d} \Sigma .
$$

Finally, (3) and (6) open the door to Weissman type estimators (Weissman, 1978) for extreme geometric quantiles. Indeed, let $\lambda \uparrow 1$ and $\lambda^{\prime} \uparrow 1$ such that $(1-\lambda) /\left(1-\lambda^{\prime}\right) \rightarrow c$ with $0<c<1$. Then, (6) entails that

$$
\frac{\|q(\lambda u)\|}{\left\|q\left(\lambda^{\prime} u\right)\right\|}=\left(\frac{1-\lambda^{\prime}}{1-\lambda}\right)^{1 / \min (2, \alpha)}(1+o(1))
$$

and consequently, from (3), the following asymptotic expansion holds:

$$
q(\lambda u)=\left(\frac{1-\lambda^{\prime}}{1-\lambda}\right)^{1 / \min (2, \alpha)} q\left(\lambda^{\prime} u\right)(1+\mathrm{o}(1))
$$

As in the univariate case, extreme geometric quantiles of large orders can therefore be deduced from extreme quantiles of smaller orders using an extrapolation factor. The estimation of an extreme geometric quantile $q(\lambda u)$, for $\lambda$ arbitrarily close to 1 , could thus be based on the estimation of an "intermediate" geometric quantile $q\left(\lambda^{\prime} u\right)$ for which $\lambda^{\prime} \uparrow 1$ slowly enough, and on the estimation of the index of regular variation $\alpha$. This is the principle of the Weissman estimator. A possible estimate of an intermediate quantile $q\left(\lambda_{n}^{\prime} u\right)$, with $\lambda_{n}^{\prime} \uparrow 1$ and $u \in S^{d-1}$, is obtained by considering the sample counterpart of the minimization problem that defines $q\left(\lambda_{n}^{\prime} u\right)$,

$$
q\left(\lambda_{n}^{\prime} u\right)=\underset{q \in \mathbb{R}^{d}}{\arg \min } \mathbb{E}(\|X-q\|-\|X\|)-\lambda_{n}^{\prime}\langle u, q\rangle,
$$

namely

$$
\widehat{q}_{n}\left(\lambda_{n}^{\prime} u\right)=\underset{q \in \mathbb{R}^{d}}{\arg \min } \frac{1}{n}\left(\sum_{i=1}^{n}\left\|X_{i}-q\right\|-\left\|X_{i}\right\|\right)-\lambda_{n}^{\prime}\langle u, q\rangle,
$$

where $\left(X_{1}, \ldots, X_{n}\right)$ is a sample of independent and identically distributed random vectors. This is a welldefined problem which almost surely admits a unique solution in our framework, see Chaudhuri (1996). Besides, the objective (random) function is almost surely finite, convex and continuous. A possible idea to study the asymptotic distribution of $\widehat{q}_{n}\left(\lambda_{n}^{\prime} u\right)$ is to use convex stochastic optimization techniques such as the results of Geyer (1996) and Knight (1999). Of course, we should expect that this estimate of $q\left(\lambda_{n}^{\prime} u\right)$ will only be consistent provided $\lambda_{n}^{\prime} \uparrow 1$ slowly enough. A detailed study of the properties of such an estimator is beyond the scope of this paper. Similarly, the estimation of $\alpha$ could be addressed in future research. 


\section{$3 \quad$ Numerical illustrations}

For the sake of illustration, we focus on the bidimensional case $d=2$ : it is assumed that $X$ follows a bivariate elliptically-contoured $\operatorname{Pareto}(\alpha, \Sigma)$ distribution, with probability density function $f(x)=$ $C_{\alpha}\left(x^{\prime} \Sigma^{-1} x\right)^{(-2-\alpha) / 2} \mathbb{1}_{[1, \infty)}\left(x^{\prime} \Sigma^{-1} x\right)$, where $C_{\alpha}>0$ is an appropriate normalizing constant. It is then straightforward to show that $f$ belongs to model $\left(M_{\alpha}^{\prime}\right)$, with $Q(x)=\left(x^{\prime} \Sigma^{-1} x\right)^{(-2-\alpha) / 2}$ and $V(t)=$ $C_{\alpha} t^{-\alpha} \mathbb{1}_{[1, \infty)}(t)$.

Let $u \in S^{1}$. Following the results of Section 2, one can obtain asymptotic expansions of the extreme quantile $q(\lambda u)$ as $\lambda \uparrow 1$. In the case $\alpha<2$, Theorem 1 yields

$$
q(\lambda u)=\left(\frac{C_{\alpha}}{1-\lambda} \int_{\mathbb{R}^{d}}\left(1+\frac{\langle y-u, u\rangle}{\|y-u\|}\right) Q(y) d y\right)^{1 / \alpha} u(1+\mathrm{o}(1)) \text { as } \lambda \uparrow 1 .
$$

When $\alpha=2$, let $h$ be the inverse of the function $t \mapsto t^{2} / \log (t)$ on $[\sqrt{e}, \infty)$. Corollary 2 then entails

$$
q(\lambda u)=h\left(\frac{\pi(\operatorname{det} \Sigma)^{1 / 2}\left(\operatorname{tr} \Sigma-u^{\prime} \Sigma u\right)}{2(1-\lambda)}\right) u(1+\mathrm{o}(1)) \text { as } \lambda \uparrow 1 .
$$

In particular, from Lemma $2,\|q(\lambda u)\|$ is of asymptotic order $(1-\lambda)^{-1 / 2}(-\log (1-\lambda))^{1 / 2}$. Finally, in the case $\alpha>2$, Proposition 1 yields

$$
q(\lambda u)=\left(\frac{\operatorname{tr} M-u^{\prime} M u}{2(1-\lambda)}\right)^{1 / 2} u(1+o(1)) \text { as } \lambda \uparrow 1, \quad \text { with } \quad M=\pi \frac{C_{\alpha}}{\alpha-2}(\operatorname{det} \Sigma)^{1 / 2} \Sigma .
$$

Letting $u_{\theta}=(\cos \theta, \sin \theta) \in S^{1}$, our goal is to compare the iso-quantile curves $\mathcal{C}_{\lambda}=\left\{q\left(\lambda u_{\theta}\right), \theta \in[0,2 \pi)\right\}$ computed by minimizing (1) numerically to the asymptotic ones, i.e. approximated using equations (9), (10) and (11). The numerical versions of the iso-quantile curves should be considered as the ground truth here. Results are displayed on Figures 2-6, for $\alpha \in\{1.3,1.5,1.7,1.9,2,2.1,2.3,2.5,3,4\}$ and $\lambda=0.995$ in the particular case where $\Sigma=\operatorname{diag}\left(\sigma_{1}^{2}, \sigma_{2}^{2}\right)$ with $\sigma_{1}=2$ and $\sigma_{2}=1 / 2$. These choices yield $C_{\alpha}=$ $\alpha /\left(2 \sigma_{1} \sigma_{2} \pi\right)=\alpha / 2 \pi$.

One can see that the asymptotic approximation works best when $|\alpha-2|$ is large, e.g. greater than 0.5. When $\alpha<2$, a possible explanation for this phenomenon lies in the proof of Theorem 1. Equations (46) and (49) imply that the error terms in the asymptotic equivalent of $V(\|q(\lambda u)\|)$ are actually of order $\left[\|q(\lambda u)\|^{2} V(\|q(\lambda u)\|)\right]^{-1}$ when $1<\alpha<2$. Moreover, from Theorem 1, the norm of the extreme quantile $\|q(\lambda u)\|$ behaves roughly like $(1-\lambda)^{-1 / \alpha}$ as $\lambda \uparrow 1$, so that the error term behaves roughly like $(1-\lambda)^{-1+2 / \alpha}$, whose convergence to 0 becomes slower as $\alpha$ approaches 2 .

Let us also remark that, for $\alpha=2$, the overall shape of the iso-quantile curve obtained by (10) is very similar to that of the curve obtained with (11) when $\alpha>2$. This may be seen as a consequence of Corollary 2 and of the link (8) between the matrices $\Sigma$ and $M$. The overall shape of the contour line with the asymptotic equivalent is different from that of the numerically approximated curve for $\alpha \geq 2$ though. In this case, the shape of the asymptotic contour curve is controlled by the function $\varphi: u \mapsto \operatorname{tr} \Sigma-u^{\prime} \Sigma u$, 
see (10) and (11). It is straightforward to see that the global maximum of the function $\varphi$ on $S^{d-1}$ is reached at a unit eigenvector $u_{\min }$ of $\Sigma$ associated with its smallest eigenvalue $\lambda_{\min }>0$. In particular, for $\alpha>2$, the norm of an extreme geometric quantile is asymptotically the largest in the direction where the variance is the smallest; in our example it is the largest in the direction of the $y$-axis. Similarly, the global minimum of $\varphi$ is reached at a unit eigenvector $u_{\max }$ of $\Sigma$ associated with its largest eigenvalue $\lambda_{\max }>0$, that is, in the direction of the $x$-axis here. This is why the asymptotic approximation to the geometric quantile contour curve has a distinctive "8" shape. The shape of the numerical approximation to the extreme geometric quantile contour curve is more oval, i.e. it is farther from the true value of the geometric quantile in the direction of the $x$-axis; a partial explanation lies again in the error terms. When $\alpha>3$, it is a consequence of Lemmas 4 and 5 in Girard and Stupfler (2015) that the error term in the approximation of the magnitude $\|q(\lambda u)\|$ of an extreme geometric quantile by its asymptotic equivalent is proportional to $\|q(\lambda u)\|^{-1}$. In other words, the approximation is best when the magnitude of the extreme geometric quantile is the highest. Another element is certainly given by considering the numerical problem defining the geometric quantile for $\alpha \in[1.5,2.5]$; one can argue that the numerical problems are close when $\alpha$ varies in this range and thus the true geometric quantile contours should also be close, which is supported by our simulation study. As a consequence, the shape of the true extreme geometric quantile curve should evolve rather slowly as $\alpha$ varies. By contrast, the form of the asymptotic equivalent changes from $\alpha<2$ to $\alpha=2$ and from $\alpha=2$ to $\alpha>2$, with a drastically different shape from the first form to the second one and slow convergence rates around $\alpha=2$. Both these reasons give an explanation for the differences in shape between the two curves.

\section{Proofs}

This section is organized as follows. Paragraph 4.1 provides two preliminary analytical lemmas. Paragraph 4.2 establishes some properties of multivariate regularly varying functions. Paragraphs 4.3, 4.4 and 4.5 are dedicated to the proofs of the main results: respectively Theorem 1, Theorem 2 and Corollary 2 .

\subsection{Preliminary results}

The first lemma is an analytical result which will reveal useful in the proof of Theorem 2 .

Lemma 1. Let $h_{1}, h_{2}:(1, \infty) \rightarrow \mathbb{R}$ be two nonnegative functions such that $h_{1}(t) \rightarrow \infty$ and $h_{2}(t) \rightarrow \infty$ as $t \rightarrow \infty$. Then, for any $\beta>0$, there exists a real function $\varepsilon:(1, \infty) \rightarrow[0, \infty)$ such that $\varepsilon(t) \rightarrow 0$, $t \varepsilon(t) \rightarrow \infty$,

$$
\varepsilon^{\beta}(t) h_{1}(t \varepsilon(t)) \rightarrow \infty \quad \text { and } \quad \frac{h_{2}(t \varepsilon(t))}{|\log \varepsilon(t)|} \rightarrow \infty \quad \text { as } t \rightarrow \infty .
$$

Proof of Lemma 1. Let us define the function $\varepsilon$ as

$$
\varepsilon(t)=\max \left(\frac{1}{\sqrt{t}},\left(\inf _{u \geq \sqrt{t}} h_{1}(u)\right)^{-1 / 2 \beta}, \exp \left(-\left[\inf _{u \geq \sqrt{t}} h_{2}(u)\right]^{1 / 2}\right)\right) .
$$


Then, clearly, $\varepsilon(t) \rightarrow 0, t \varepsilon(t) \geq \sqrt{t} \rightarrow \infty$ and

$$
\varepsilon^{\beta}(t) h_{1}(t \varepsilon(t)) \geq\left(\inf _{u \geq \sqrt{t}} h_{1}(u)\right)^{-1 / 2} \times \inf _{u \geq t \varepsilon(t)} h_{1}(u) \geq\left(\inf _{u \geq \sqrt{t}} h_{1}(u)\right)^{1 / 2} \rightarrow \infty \text { as } t \rightarrow \infty .
$$

Similarly, since the logarithm function is increasing, one has for $t$ large enough

$$
\frac{1}{|\log \varepsilon(t)|}=\frac{1}{-\log \varepsilon(t)} \geq\left(\inf _{u \geq \sqrt{t}} h_{2}(u)\right)^{-1 / 2}
$$

and therefore

$$
\frac{h_{2}(t \varepsilon(t))}{|\log \varepsilon(t)|} \geq\left(\inf _{u \geq \sqrt{t}} h_{2}(u)\right)^{-1 / 2} \times \inf _{u \geq t \varepsilon(t)} h_{2}(u) \geq\left(\inf _{u \geq \sqrt{t}} h_{2}(u)\right)^{1 / 2} \rightarrow \infty \text { as } t \rightarrow \infty
$$

which proves the result.

Lemma 2 is an analytical result needed to illustrate Theorem 2. It is well-known that the inverse of a regularly varying function with index $a>0$ is regularly varying with index $1 / a$ (see e.g. Theorem 1.5.12 p.28 in Bingham et al., 1987). Lemma 2 provides an asymptotic equivalent of the inverse for a particular class of functions.

Lemma 2. Let $a>0, b \in \mathbb{R}$ and define $g_{a, b}: t \mapsto t^{a}(\log t)^{b}$ on $(1, \infty)$. Then $g_{a, b}$ has an inverse $h_{a, b}$ on a neighborhood of infinity which is such that

$$
h_{a, b}(t)=a^{b / a} \frac{t^{1 / a}}{(\log t)^{b / a}}(1+\mathrm{o}(1)) \text { as } t \rightarrow \infty .
$$

Proof of Lemma 2. Let us remark that $g_{a, b}$ is continuously differentiable on $(1, \infty)$ with derivative $g_{a, b}^{\prime}(t)=t^{a-1}(\log t)^{b-1}[a \log t+b]$ for all $t>1$. Clearly, $g_{a, b}^{\prime}(t)$ is positive for $t>e^{-b / a}$ and thus $g_{a, b}$ has an inverse on a neighborhood of infinity denoted by $h_{a, b}$. For all $t$ large enough, one has

$$
t=\left[h_{a, b}(t)\right]^{a}\left[\log \left(h_{a, b}(t)\right)\right]^{b} \Rightarrow h_{a, b}(t)=t^{1 / a}\left[\log \left(h_{a, b}(t)\right)\right]^{-b / a},
$$

and an iterated use of (12) entails

$$
h_{a, b}(t)=t^{1 / a}\left[\log \left(t^{1 / a}\left[\log \left(h_{a, b}(t)\right)\right]^{-b / a}\right)\right]^{-b / a} \Rightarrow h_{a, b}(t)=a^{b / a} \frac{t^{1 / a}}{(\log t)^{b / a}}\left(1-b \frac{\log \log h_{a, b}(t)}{\log t}\right)^{-b / a} .
$$

Since $g_{a, b}$ is increasing on a neighborhood of infinity and tends to infinity at infinity, so does $h_{a, b}$. Taking logarithms in (12) entails $\log t=a \log h_{a, b}(t)+b \log \log h_{a, b}(t)$ and consequently

$$
\log h_{a, b}(t)=\frac{1}{a} \log t(1+\mathrm{o}(1)) \text { as } t \rightarrow \infty .
$$

This yields

$$
\frac{\log \log h_{a, b}(t)}{\log t} \rightarrow 0 \text { as } t \rightarrow \infty .
$$

Plugging (14) in (13) completes the proof. 


\subsection{Auxiliary results on multivariate regular variation}

Let us start with some useful consequences of condition $\left(M_{\alpha}\right)$.

Lemma 3. Assume that $\left(M_{\alpha}\right)$ holds for some $\alpha>0$. Then,

(i) $Q$ is a homogeneous continuous function of degree $-d-\alpha$ on $\mathbb{R}^{d} \backslash\{0\}$;

(ii) One has $f(y)=\|y\|^{-d} V(\|y\|) Q(y /\|y\|)(1+\theta(y))$ where $\theta(y) \rightarrow 0$ as $\|y\| \rightarrow \infty$.

Proof of Lemma 3. To prove (i), it is enough to note that $Q$ is continuous on $S^{d-1}$ as a direct consequence of $\left(M_{\alpha}\right)$, while the homogeneity follows from the convergences

$$
\frac{f(t(a y))}{t^{-d} V(t)} \rightarrow Q(a y) \text { and } \frac{f(t(a y))}{t^{-d} V(t)}=a^{-d} \frac{V(a t)}{V(t)} \frac{f((a t) y)}{(a t)^{-d} V(a t)} \rightarrow a^{-d-\alpha} Q(y) \text { as } t \rightarrow \infty,
$$

valid for every $a>0$ and $y \neq 0$. To obtain (ii), observe that

$$
\left|\frac{f(y)}{\|y\|^{-d} V(\|y\|)}-Q\left(\frac{y}{\|y\|}\right)\right| \leq \sup _{w \in S^{d-1}}\left|\frac{f(\|y\| w)}{\|y\|^{-d} V(\|y\|)}-Q(w)\right| .
$$

Therefore, condition $\left(M_{\alpha}\right)$ entails

$$
f(y)=\|y\|^{-d} V(\|y\|)\left(Q(y /\|y\|)+\theta^{*}(y)\right) \text { with } \theta^{*}(y) \rightarrow 0 \text { as }\|y\| \rightarrow \infty .
$$

Since $Q$ is positive and continuous on the compact set $S^{d-1}$, it is bounded from below by a positive constant on $S^{d-1}$ and thus

$$
f(y)=\|y\|^{-d} V(\|y\|) Q(y /\|y\|)(1+\theta(y)) \text { with } \theta(y):=\frac{\theta^{*}(y)}{Q(y /\|y\|)} \rightarrow 0 \text { as }\|y\| \rightarrow \infty .
$$

The result follows.

The second lemma is a slightly stronger version of some uniform convergence results proved in Lemma 1 of Cai et al. (2011).

Lemma 4. Assume that $\left(M_{\alpha}\right)$ holds for some $\alpha>0$. Then for every $\delta, \varepsilon>0$, we have that

$$
\sup _{\|y\| \geq \varepsilon}\|y\|^{d+\alpha-\delta}\left|\frac{f(t y)}{t^{-d} V(t)}-Q(y)\right| \rightarrow 0 \quad \text { and } \sup _{0<\|y\| \leq \varepsilon}\|y\|^{d+\alpha+\delta}\left|\frac{f(t y)}{t^{-d} V(t)}-Q(y)\right| \rightarrow 0 \quad \text { as } \quad t \rightarrow \infty \text {. }
$$

Proof of Lemma 4. To prove the first convergence, use the triangle inequality and the homogeneity of $Q$ to obtain for every $y \neq 0$

$$
\begin{aligned}
\|y\|^{d+\alpha-\delta}\left|\frac{f(t y)}{t^{-d} V(t)}-Q(y)\right| & \leq\|y\|^{-\delta}\left|\frac{f(t\|y\|(y /\|y\|))}{(t\|y\|)^{-d} V(t\|y\|)}-Q\left(\frac{y}{\|y\|}\right)\right| \\
& +\frac{f(t\|y\|(y /\|y\|))}{(t\|y\|)^{-d} V(t\|y\|)}\left|\frac{(t\|y\|)^{\alpha-\delta} V(t\|y\|)}{t^{\alpha-\delta} V(t)}-\|y\|^{-\delta}\right| .
\end{aligned}
$$

For every $y$ such that $\|y\| \geq \varepsilon>0$, we have on the one hand

$$
\|y\|^{-\delta}\left|\frac{f(t\|y\|(y /\|y\|))}{(t\|y\|)^{-d} V(t\|y\|)}-Q\left(\frac{y}{\|y\|}\right)\right| \leq \varepsilon^{-\delta} \sup _{T \geq t \varepsilon} \sup _{w \in S^{d-1}}\left|\frac{f(T w)}{T^{-d} V(T)}-Q(w)\right| \rightarrow 0 \text { as } t \rightarrow \infty .
$$


On the other hand, for $t$ large enough, using Lemma 3(ii) and the boundedness of the continuous function $Q$ on the compact set $S^{d-1}$ entails:

$$
\frac{f(t\|y\|(y /\|y\|))}{(t\|y\|)^{-d} V(t\|y\|)}\left|\frac{(t\|y\|)^{\alpha-\delta} V(t\|y\|)}{t^{\alpha-\delta} V(t)}-\|y\|^{-\delta}\right|=\mathrm{O}\left(\sup _{a \geq \varepsilon}\left|\frac{(a t)^{\alpha-\delta} V(a t)}{t^{\alpha-\delta} V(t)}-a^{-\delta}\right|\right) .
$$

Remarking that $t \mapsto t^{\alpha-\delta} V(t)$ is regularly varying at infinity with index $-\delta<0$, a uniform convergence property (see e.g. Theorem 1.5.2 p.22 in Bingham et al., 1987) yields

$$
\sup _{\|y\| \geq \varepsilon} \frac{f(t\|y\|(y /\|y\|))}{(t\|y\|)^{-d} V(t\|y\|)}\left|\frac{(t\|y\|)^{\alpha-\delta} V(t\|y\|)}{t^{\alpha-\delta} V(t)}-\|y\|^{-\delta}\right| \rightarrow 0 \quad \text { as } \quad t \rightarrow \infty .
$$

Combining (15), (16) and (17) yields the first part of the result.

We now prove the second convergence. Pick an arbitrary $\eta>0$ and let $t_{0}>0$ be such that

$$
\forall t>t_{0}, \sup _{w \in S^{d-1}}\left|\frac{f(t w)}{t^{-d} V(t)}-Q(w)\right|<\frac{\eta}{2 \varepsilon^{\delta}} .
$$

Define a function $\tilde{V}$ by $\widetilde{V}(t)=1$ if $0 \leq t \leq t_{0}$ and $\widetilde{V}(t)=V(t)$ otherwise. For all $t>t_{0}$, we have that

$$
\begin{aligned}
\|y\|^{d+\alpha+\delta}\left|\frac{f(t y)}{t^{-d} V(t)}-Q(y)\right| & \leq\|y\|^{\delta}\left|\frac{f(t\|y\|(y /\|y\|))}{(t\|y\|)^{-d} \widetilde{V}(t\|y\|)}-Q\left(\frac{y}{\|y\|}\right)\right| \\
& +\frac{f(t\|y\|(y /\|y\|))}{(t\|y\|)^{-d} \widetilde{V}(t\|y\|)}\left|\frac{(t\|y\|)^{\alpha+\delta} \widetilde{V}(t\|y\|)}{t^{\alpha+\delta} \widetilde{V}(t)}-\|y\|^{\delta}\right| .
\end{aligned}
$$

Inequality (18) entails

$$
\|y\|^{\delta}\left|\frac{f(t\|y\|(y /\|y\|))}{(t\|y\|)^{-d} \widetilde{V}(t\|y\|)}-Q\left(\frac{y}{\|y\|}\right)\right| \mathbb{1}_{\left\{t_{0} / t<\|y\| \leq \varepsilon\right\}} \leq \frac{\eta}{2} .
$$

Moreover,

$$
\|y\|^{\delta}\left|\frac{f(t\|y\|(y /\|y\|))}{(t\|y\|)^{-d} \widetilde{V}(t\|y\|)}-Q\left(\frac{y}{\|y\|}\right)\right| \mathbb{1}_{\left\{0<\|y\| \leq t_{0} / t\right\}} \leq\left(\frac{t_{0}}{t}\right)^{\delta}\left(\sup _{\|z\| \leq t_{0}} \frac{\|z\|^{d} f(z)}{\widetilde{V}(\|z\|)}+\sup _{w \in S^{d-1}} Q(w)\right) .
$$

Since $y \mapsto\|y\|^{d} f(y)$ is bounded on any compact neighborhood of 0 and $\widetilde{V}$ is equal to 1 on $\left[0, t_{0}\right]$ and $Q$ is bounded on $S^{d-1}$, the right-hand side above is finite. One thus obtains for $t$ large enough

$$
\|y\|^{\delta}\left|\frac{f(t\|y\|(y /\|y\|))}{(t\|y\|)^{-d} \widetilde{V}(t\|y\|)}-Q\left(\frac{y}{\|y\|}\right)\right| \mathbb{1}_{\left\{0<\|y\| \leq t_{0} / t\right\}} \leq \frac{\eta}{2} .
$$

Combining (20) and (21), it becomes clear that

$$
\sup _{0<\|y\| \leq \varepsilon}\|y\|^{\delta}\left|\frac{f(t\|y\|(y /\|y\|))}{(t\|y\|)^{-d} \widetilde{V}(t\|y\|)}-Q\left(\frac{y}{\|y\|}\right)\right| \rightarrow 0 \text { as } t \rightarrow \infty .
$$

Finally, let us remark that (18) entails

$$
\sup _{0<\|y\| \leq \varepsilon} \frac{f(t\|y\|(y /\|y\|))}{(t\|y\|)^{-d} \widetilde{V}(t\|y\|)} \leq \sup _{\|z\| \leq t_{0}} \frac{\|z\|^{d} f(z)}{\widetilde{V}(\|z\|)}+2 \sup _{w \in S^{d-1}} Q(w)+\frac{\eta}{2 \varepsilon^{\delta}}<\infty
$$


and therefore, since $t \mapsto t^{\alpha+\delta} V(t)$ is regularly varying at infinity with index $\delta>0$ and bounded on any neighborhood of 0, a uniform convergence result (see e.g. Theorem 1.5.2 p.22 in Bingham et al., 1987) yields, as $t \rightarrow \infty$,

$$
\sup _{0<\|y\| \leq \varepsilon} \frac{f(t\|y\|(y /\|y\|))}{(t\|y\|)^{-d} \widetilde{V}(t\|y\|)}\left|\frac{(t\|y\|)^{\alpha+\delta} \widetilde{V}(t\|y\|)}{t^{\alpha+\delta} \widetilde{V}(t)}-\|y\|^{\delta}\right|=\mathrm{O}\left(\sup _{0<a \leq \varepsilon}\left|\frac{(a t)^{\alpha+\delta} \widetilde{V}(a t)}{t^{\alpha+\delta} \widetilde{V}(t)}-a^{\delta}\right|\right) \rightarrow 0 .
$$

Combining (19), (22) and (23) completes the proof.

\subsection{Proof of Theorem 1}

Lemma 5 is the essential tool to prove Theorem 1(i).

Lemma 5. Let $u \in S^{d-1}$.

(i) Assume that $\left(M_{\alpha}\right)$ holds for some $\alpha \in(0,1)$. Let $v \in \mathbb{R}^{d}$ and define

$$
I(u, v)=\int_{\mathbb{R}^{d}}\left(\frac{\langle y-u, v\rangle}{\|y-u\|}+\langle u, v\rangle\right) Q(y) d y .
$$

Then $I(u, v)$ is well-defined, finite and

$$
\frac{1}{V(\|q(\lambda u)\|)}\left\langle\lambda u-\frac{q(\lambda u)}{\|q(\lambda u)\|}, v\right\rangle \rightarrow-I(u, v) \quad \text { as } \quad \lambda \uparrow 1 .
$$

(ii) Assume that $\left(M_{1}\right)$ holds. Then, for any $\varepsilon>0$,

$$
\frac{1}{V(\|q(\lambda u)\|)}\left\langle\lambda u-\frac{q(\lambda u)}{\|q(\lambda u)\|}, v\right\rangle=\mathrm{o}\left(\|q(\lambda u)\|^{\varepsilon}\right) \quad \text { as } \quad \lambda \uparrow 1 \text {. }
$$

Proof of Lemma 5. (i) The Cauchy-Schwarz inequality entails, for all $y \in \mathbb{R}^{d}$ and $w \in S^{d-1}$,

$$
\left|\frac{\langle y-w, v\rangle}{\|y-w\|}+\langle w, v\rangle\right| \leq\left\|\frac{y-w}{\|y-w\|}+w\right\|\|v\|
$$

Besides,

$$
\left\|\frac{y-w}{\|y-w\|}+w\right\|^{2}=2\left(1+\left\langle\frac{y-w}{\|y-w\|}, w\right\rangle\right)=\frac{2(\|y-w\|+\langle y-w, w\rangle)}{\|y-w\|}
$$

so that

$$
\left\|\frac{y-w}{\|y-w\|}+w\right\|^{2}=\frac{2\left(\|y-w\|^{2}-\langle y-w, w\rangle^{2}\right)}{\|y-w\|(\|y-w\|-\langle y-w, w\rangle)} .
$$

The numerator of the right-hand side can be bounded from above as follows:

$$
\|y-w\|^{2}-\langle y-w, w\rangle^{2}=\|y\|^{2}-\langle y, w\rangle^{2} \leq\|y\|^{2} .
$$

If moreover $\|y\|<\|w\|=1$, then the denominator can be controlled by applying both the Cauchy-Schwarz and reverse triangle inequalities:

$$
\|y-w\|(\|y-w\|-\langle y-w, w\rangle)=\|y-w\|^{2}\left(1+\|y-w\|-\frac{\langle y-w, y\rangle}{\|y-w\|}\right) \geq 2(1-\|y\|)^{3} .
$$


As a consequence, if $\mathcal{B}$ is the ball centered at the origin having radius $1 / 2$, then

$$
\forall y \in \mathcal{B},\left|\frac{\langle y-w, v\rangle}{\|y-w\|}+\langle w, v\rangle\right| \leq 2\|v\|\|y\|,
$$

and the homogeneity property of $Q$ yields

$$
\left|\frac{\langle y-w, v\rangle}{\|y-w\|}+\langle w, v\rangle\right| Q(y) \mathbb{1}_{\mathcal{B}}(y) \leq 2\|v\|\|y\|^{-(d-1+\alpha)} Q\left(\frac{y}{\|y\|}\right) \mathbb{1}_{\mathcal{B}}(y) .
$$

The right-hand side of this inequality defines an integrable function in a neighborhood of 0 because $\alpha \in(0,1)$. Besides, the Cauchy-Schwarz inequality entails for all $y \in \mathbb{R}^{d}$ and $w \in S^{d-1}$

$$
\left|\frac{\langle y-w, v\rangle}{\|y-w\|}+\langle w, v\rangle\right| \leq 2\|v\| .
$$

Consequently, denoting by $\mathcal{B}^{c}$ the complement of $\mathcal{B}$, it follows that

$$
\left|\frac{\langle y-w, v\rangle}{\|y-w\|}+\langle w, v\rangle\right| Q(y) \mathbb{1}_{\mathcal{B}^{c}}(y) \leq 2\|v\|\|y\|^{-(d+\alpha)} Q\left(\frac{y}{\|y\|}\right) \mathbb{1}_{\mathcal{B}^{c}}(y)
$$

where the right-hand side defines an integrable function in a neighborhood of infinity since $\alpha>0$. Combining (25) and (27) with $w=u$ shows that $I(u, v)$ is finite. The characterization of the geometric quantile (2) yields

$$
\lambda u+\int_{\mathbb{R}^{d}} \frac{x-q(\lambda u)}{\|x-q(\lambda u)\|} f(x) d x=0
$$

or equivalently

$$
\begin{aligned}
I_{1}(\lambda)+I_{2}(\lambda) & =-\frac{1}{V(\|q(\lambda u)\|)}\left\langle\lambda u-\frac{q(\lambda u)}{\|q(\lambda u)\|}, v\right\rangle \\
\text { with } I_{1}(\lambda) & =\int_{\mathcal{B}}\left(\left\|y-\frac{q(\lambda u)}{\|q(\lambda u)\|}\right\|^{-1}\left\langle y-\frac{q(\lambda u)}{\|q(\lambda u)\|}, v\right\rangle+\left\langle\frac{q(\lambda u)}{\|q(\lambda u)\|}, v\right\rangle\right) \frac{f(\|q(\lambda u)\| y)}{\|q(\lambda u)\|^{-d} V(\|q(\lambda u)\|)} d y \\
\text { and } I_{2}(\lambda) & =\int_{\mathcal{B}^{c}}\left(\left\|y-\frac{q(\lambda u)}{\|q(\lambda u)\|}\right\|^{-1}\left\langle y-\frac{q(\lambda u)}{\|q(\lambda u)\|}, v\right\rangle+\left\langle\frac{q(\lambda u)}{\|q(\lambda u)\|}, v\right\rangle\right) \frac{f(\|q(\lambda u)\| y)}{\|q(\lambda u)\|^{-d} V(\|q(\lambda u)\|)} d y .
\end{aligned}
$$

Let us also introduce

$$
\begin{aligned}
I_{1}^{\prime}(\lambda) & =\int_{\mathcal{B}}\left(\left\|y-\frac{q(\lambda u)}{\|q(\lambda u)\|}\right\|^{-1}\left\langle y-\frac{q(\lambda u)}{\|q(\lambda u)\|}, v\right\rangle+\left\langle\frac{q(\lambda u)}{\|q(\lambda u)\|}, v\right\rangle\right) Q(y) d y \\
\text { and } I_{2}^{\prime}(\lambda) & =\int_{\mathcal{B}^{c}}\left(\left\|y-\frac{q(\lambda u)}{\|q(\lambda u)\|}\right\|^{-1}\left\langle y-\frac{q(\lambda u)}{\|q(\lambda u)\|}, v\right\rangle+\left\langle\frac{q(\lambda u)}{\|q(\lambda u)\|}, v\right\rangle\right) Q(y) d y .
\end{aligned}
$$

To obtain the asymptotic behavior of $I_{1}(\lambda)$, we first deduce from (24) that:

$$
\left|I_{1}(\lambda)-I_{1}^{\prime}(\lambda)\right| \leq 2\|v\| \int_{\mathcal{B}}\left\{\|y\|^{d+\alpha+\delta}\left|\frac{f(\|q(\lambda u)\| y)}{\|q(\lambda u)\|^{-d} V(\|q(\lambda u)\|)}-Q(y)\right|\right\} \frac{d y}{\|y\|^{d-1+\alpha+\delta}}
$$

where $\delta=(1-\alpha) / 2>0$ is such that $\alpha+\delta<1$. Let us note that since $y \mapsto\|y\|^{-(d-1+\alpha+\delta)}$ is an integrable function in a neighborhood of 0 , Lemma 4 entails that $I_{1}(\lambda)-I_{1}^{\prime}(\lambda) \rightarrow 0$ as $\lambda \uparrow 1$. Recalling (25) with $w$ replaced by $q(\lambda u) /\|q(\lambda u)\|$ and applying (3) together with the dominated convergence theorem, we obtain:

$$
I_{1}(\lambda) \rightarrow \int_{\mathcal{B}}\left(\frac{\langle y-u, v\rangle}{\|y-u\|}+\langle u, v\rangle\right) Q(y) d y \text { as } \lambda \uparrow 1 .
$$


Let us now focus on $I_{2}(\lambda)$ : from $(26)$, it follows that

$$
\left|I_{2}(\lambda)-I_{2}^{\prime}(\lambda)\right| \leq 2\|v\| \int_{\mathcal{B}^{c}}\left\{\|y\|^{d+\alpha / 2}\left|\frac{f(\|q(\lambda u)\| y)}{\|q(\lambda u)\|^{-d} V(\|q(\lambda u)\|)}-Q(y)\right|\right\} \frac{d y}{\|y\|^{d+\alpha / 2}} .
$$

Since $\alpha>0$, the function $y \mapsto\|y\|^{d+\alpha / 2}$ is integrable in a neighborhood of infinity. Lemma 4 thus entails that $I_{2}(\lambda)-I_{2}^{\prime}(\lambda) \rightarrow 0$ as $\lambda \uparrow 1$. Recalling (27) with $w$ replaced by $q(\lambda u) /\|q(\lambda u)\|$ and applying (3) together with the dominated convergence theorem, we get

$$
I_{2}(\lambda) \rightarrow \int_{\mathcal{B}^{c}}\left(\frac{\langle y-u, v\rangle}{\|y-u\|}+\langle u, v\rangle\right) Q(y) d y \text { as } \lambda \uparrow 1 .
$$

The result follows by combining (28), (29) and (30).

(ii) Since $B^{d}$ is a relatively compact neighborhood of 0 , inequality (24) entails for $n$ large enough

$$
\forall x \in B^{d},\left|\frac{\langle x-q(\lambda u), v\rangle}{\|x-q(\lambda u)\|}+\left\langle\frac{q(\lambda u)}{\|q(\lambda u)\|}, v\right\rangle\right| \leq 2 \frac{\|v\|\|x\|}{\|q(\lambda u)\|} \leq 2 \frac{\|v\|}{\|q(\lambda u)\|}
$$

and therefore

$$
\int_{B^{d}}\left(\frac{\langle x-q(\lambda u), v\rangle}{\|x-q(\lambda u)\|}+\left\langle\frac{q(\lambda u)}{\|q(\lambda u)\|}, v\right\rangle\right) f(x) d x=\mathrm{O}\left(\|q(\lambda u)\|^{-1}\right) .
$$

Let further $\mathcal{A}(\lambda)$ be the annulus $\left\{y \in \mathbb{R}^{d} \mid 1 /\|q(\lambda u)\|<\|y\| \leq 1 / 2\right\} \subset \mathcal{B}$. Similarly to what was done in the proof of (i), equation (2) and a change of variables entail

$$
\begin{aligned}
I_{2}(\lambda)+I_{3}(\lambda) & =-\frac{1}{V(\|q(\lambda u)\|)}\left\langle\lambda u-\frac{q(\lambda u)}{\|q(\lambda u)\|}, v\right\rangle+\mathrm{O}\left([\|q(\lambda u)\| V(\|q(\lambda u)\|)]^{-1}\right) \\
\text { with } I_{3}(\lambda) & =\int_{\mathcal{A}(\lambda)}\left(\left\|y-\frac{q(\lambda u)}{\|q(\lambda u)\|}\right\|^{-1}\left\langle y-\frac{q(\lambda u)}{\|q(\lambda u)\|}, v\right\rangle+\left\langle\frac{q(\lambda u)}{\|q(\lambda u)\|}, v\right\rangle\right) \frac{f(\|q(\lambda u)\| y)}{\|q(\lambda u)\|^{-d} V(\|q(\lambda u)\|)} d y
\end{aligned}
$$

where $I_{2}(\lambda)$ is defined in the proof of (i). Since $t \mapsto[t V(t)]^{-1}$ is slowly varying, Proposition 1.3.6(v) p.16 in Bingham et al. (1987) gives $[t V(t)]^{-1}=\mathrm{o}\left(t^{\varepsilon}\right)$ as $t \rightarrow \infty$ for any $\varepsilon>0$. Recall further that (30) was actually also true for $\alpha=1$, so that

$$
\frac{1}{V(\|q(\lambda u)\|)}\left\langle\lambda u-\frac{q(\lambda u)}{\|q(\lambda u)\|}, v\right\rangle=-I_{3}(\lambda)+\mathrm{o}\left(\|q(\lambda u)\|^{\varepsilon}\right) .
$$

Let us now turn to the control of $I_{3}(\lambda)$ and introduce

$$
I_{3}^{\prime}(\lambda)=\int_{\mathcal{A}(\lambda)}\left(\left\|y-\frac{q(\lambda u)}{\|q(\lambda u)\|}\right\|^{-1}\left\langle y-\frac{q(\lambda u)}{\|q(\lambda u)\|}, v\right\rangle+\left\langle\frac{q(\lambda u)}{\|q(\lambda u)\|}, v\right\rangle\right) Q(y) d y .
$$

From (24), it follows that as $\lambda \uparrow 1$,

$$
\begin{aligned}
\left|I_{3}(\lambda)-I_{3}^{\prime}(\lambda)\right| & \leq 2\|v\| \int_{\mathcal{A}(\lambda)}\left\{\|y\|^{d+1+\varepsilon}\left|\frac{f(\|q(\lambda u)\| y)}{\|q(\lambda u)\|^{-d} V(\|q(\lambda u)\|)}-Q(y)\right|\right\} \frac{d y}{\|y\|^{d+\varepsilon}} \\
& =\mathrm{o}\left(\int_{\mathcal{A}(\lambda)} \frac{d y}{\|y\|^{d+\varepsilon}}\right)=\mathrm{o}\left(\int_{1 /\|q(\lambda u)\|}^{1} \frac{d r}{r^{1+\varepsilon}}\right)=\mathrm{o}\left(\|q(\lambda u)\|^{\varepsilon}\right),
\end{aligned}
$$

in view of Lemma 4 and after switching to polar coordinates. Finally, $I_{3}^{\prime}(\lambda)$ is controlled using $(25)$ :

$$
I_{3}^{\prime}(\lambda) \leq 2\|v\| \int_{\mathcal{A}(\lambda)}\|y\|^{-d} Q\left(\frac{y}{\|y\|}\right) d y
$$


We can thus use the boundedness of $Q$ on $S^{d-1}$ and polar coordinates to obtain

$$
I_{3}^{\prime}(\lambda)=\mathrm{O}\left(\int_{1 /\|q(\lambda u)\|}^{1} \frac{d r}{r}\right)=\mathrm{O}(\log \|q(\lambda u)\|)=\mathrm{o}\left(\|q(\lambda u)\|^{\varepsilon}\right) \text { as } \lambda \uparrow 1 .
$$

Combining (31), (32) and (33) completes the proof.

Lemma 6 below is the key to the proof of Theorem 1(ii).

Lemma 6. Assume that $\left(M_{\alpha}\right)$ holds for some $\alpha \in(0,2)$. Let $u \in S^{d-1}$ and set

$$
J(u)=\int_{\mathbb{R}^{d}}\left(1+\frac{\langle y-u, u\rangle}{\|y-u\|}\right) Q(y) d y .
$$

Then $J(u)$ is well-defined, positive and finite, and

$$
\frac{1}{V(\|q(\lambda u)\|)}\left\langle\lambda u-\frac{q(\lambda u)}{\|q(\lambda u)\|}, \frac{q(\lambda u)}{\|q(\lambda u)\|}\right\rangle \rightarrow-J(u) \text { as } \lambda \uparrow 1 .
$$

Proof of Lemma 6. The Cauchy-Schwarz inequality yields for every $y \in \mathbb{R}^{d}$

$$
1+\frac{\langle y-u, u\rangle}{\|y-u\|} \geq 1-\|u\|=0
$$

with equality if and only if $y$ and $u$ are linearly dependent. As a consequence, $J(u)>0$. Let $\varphi$ : $\mathbb{R}^{d} \times[0, \infty) \times S^{d-1} \rightarrow \mathbb{R}$ be the nonnegative function defined by

$$
\varphi(x, r, w)=r^{2}\left(1+\frac{\langle x-r w, w\rangle}{\|x-r w\|}\right),
$$

and recall that, from Girard and Stupfler (2015, inequality (16)):

$$
\varphi(x, r, w) \mathbb{1}_{\{\|x\|<r\}} \leq\|x\|^{2} \mathbb{1}_{\{\|x\|<r\}} .
$$

Thus, using the homogeneity of $Q$ and applying (34) with $r=1$ yield for every $y \in \mathbb{R}^{d}$ and $w \in S^{d-1}$

$$
\left|1+\frac{\langle y-w, w\rangle}{\|y-w\|}\right| Q(y) \mathbb{1}_{B^{d}}(y) \leq\|y\|^{-(d-2+\alpha)} Q\left(\frac{y}{\|y\|}\right) \mathbb{1}_{B^{d}}(y)
$$

with the right-hand side of this inequality being an integrable function in a neighborhood of 0 . Besides, the Cauchy-Schwarz inequality and the homogeneity of $Q$ entail for all $y \in \mathbb{R}^{d}$ and $w \in S^{d-1}$

$$
\left|1+\frac{\langle y-w, w\rangle}{\|y-w\|}\right| Q(y) \mathbb{1}_{\mathbb{R}^{d} \backslash B^{d}}(y) \leq 2\|y\|^{-(d+\alpha)} Q\left(\frac{y}{\|y\|}\right) \mathbb{1}_{\mathbb{R}^{d} \backslash B^{d}}(y)
$$

so that the integrand in $J(u)$ is also integrable in a neighborhood of infinity. $J(u)$ is thus positive and finite. The remainder of the proof follows the lines of the proof of Lemma 5: taking account of (2) and using a change of variables, we get

$$
\begin{aligned}
J_{1}(\lambda)+J_{2}(\lambda) & =-\frac{1}{V(\|q(\lambda u)\|)}\left\langle\lambda u-\frac{q(\lambda u)}{\|q(\lambda u)\|}, \frac{q(\lambda u)}{\|q(\lambda u)\|}\right\rangle \\
\text { with } J_{1}(\lambda) & =\int_{B^{d}}\left(1+\left\|y-\frac{q(\lambda u)}{\|q(\lambda u)\|}\right\|^{-1}\left\langle y-\frac{q(\lambda u)}{\|q(\lambda u)\|}, \frac{q(\lambda u)}{\|q(\lambda u)\|}\right\rangle\right) \frac{f(\|q(\lambda u)\| y)}{\|q(\lambda u)\|^{-d} V(\|q(\lambda u)\|)} d y \\
\text { and } J_{2}(\lambda) & =\int_{\mathbb{R}^{d} \backslash B^{d}}\left(1+\left\|y-\frac{q(\lambda u)}{\|q(\lambda u)\|}\right\|^{-1}\left\langle y-\frac{q(\lambda u)}{\|q(\lambda u)\|}, \frac{q(\lambda u)}{\|q(\lambda u)\|}\right\rangle\right) \frac{f(\|q(\lambda u)\| y)}{\|q(\lambda u)\|^{-d} V(\|q(\lambda u)\|)} d y .
\end{aligned}
$$


Let us consider

$$
\begin{aligned}
J_{1}^{\prime}(\lambda) & =\int_{B^{d}}\left(1+\left\|y-\frac{q(\lambda u)}{\|q(\lambda u)\|}\right\|^{-1}\left\langle y-\frac{q(\lambda u)}{\|q(\lambda u)\|}, \frac{q(\lambda u)}{\|q(\lambda u)\|}\right\rangle\right) Q(y) d y \\
\text { and } J_{2}^{\prime}(\lambda) & =\int_{\mathbb{R}^{d} \backslash B^{d}}\left(1+\left\|y-\frac{q(\lambda u)}{\|q(\lambda u)\|}\right\|^{-1}\left\langle y-\frac{q(\lambda u)}{\|q(\lambda u)\|}, \frac{q(\lambda u)}{\|q(\lambda u)\|}\right\rangle\right) Q(y) d y .
\end{aligned}
$$

The asymptotic behavior of $J_{1}(\lambda)$ is then deduced from (34) with $r=1$ :

$$
\left|J_{1}(\lambda)-J_{1}^{\prime}(\lambda)\right| \leq \int_{B^{d}}\left\{\|y\|^{d+\alpha+\delta}\left|\frac{f(\|q(\lambda u)\| y)}{\|q(\lambda u)\|^{-d} V(\|q(\lambda u)\|)}-Q(y)\right|\right\} \frac{d y}{\|y\|^{d-2+\alpha+\delta}}
$$

where $\delta=(2-\alpha) / 2>0$ is such that $\alpha+\delta<2$. Remarking that $y \mapsto\|y\|^{-(d-2+\alpha+\delta)}$ is an integrable function in a neighborhood of 0 , Lemma 4 entails $J_{1}(\lambda)-J_{1}^{\prime}(\lambda) \rightarrow 0$ as $\lambda \uparrow 1$. In view of (35), the dominated convergence theorem leads to

$$
J_{1}(\lambda) \rightarrow \int_{B^{d}}\left(1+\frac{\langle y-u, u\rangle}{\|y-u\|}\right) Q(y) d y \text { as } \lambda \uparrow 1 .
$$

Let us now focus on $J_{2}(\lambda)$. The Cauchy-Schwarz inequality yields

$$
\left|J_{2}(\lambda)-J_{2}^{\prime}(\lambda)\right| \leq 2 \int_{\mathbb{R}^{d} \backslash B^{d}}\left\{\|y\|^{d+\alpha / 2}\left|\frac{f(\|q(\lambda u)\| y)}{\|q(\lambda u)\|^{-d} V(\|q(\lambda u)\|)}-Q(y)\right|\right\} \frac{d y}{\|y\|^{d+\alpha / 2}} .
$$

Since $y \mapsto\|y\|^{d+\alpha / 2}$ is an integrable function in a neighborhood of infinity, applying Lemma 4 shows that $J_{2}(\lambda)-J_{2}^{\prime}(\lambda) \rightarrow 0$ as $\lambda \uparrow 1$. Therefore, recalling (36) and applying the dominated convergence theorem, we get

$$
J_{2}(\lambda) \rightarrow \int_{\mathbb{R}^{d} \backslash B^{d}}\left(1+\frac{\langle y-u, u\rangle}{\|y-u\|}\right) Q(y) d y \text { as } \lambda \uparrow 1 .
$$

The result follows from (37), (38) and (39).

Proof of Theorem 1. (i) Let $\left(u, v_{1}, \ldots, v_{d-1}\right)$ be an orthonormal basis of $\mathbb{R}^{d}$. We may then write

$$
\frac{q(\lambda u)}{\|q(\lambda u)\|}-u=(b(\lambda)-1) u+\sum_{k=1}^{d-1} \beta_{k}(\lambda) v_{k}
$$

where $b(\lambda) \in \mathbb{R}$ and, for all $k \in\{1, \ldots, d-1\}$,

$$
\beta_{k}(\lambda):=\left\langle\frac{q(\lambda u)}{\|q(\lambda u)\|}, v_{k}\right\rangle=-\left\langle\lambda u-\frac{q(\lambda u)}{\|q(\lambda u)\|}, v_{k}\right\rangle .
$$

Lemma 5 entails that, for all $k \in\{1, \ldots, d-1\}$,

$$
\frac{\beta_{k}(\lambda)}{V(\|q(\lambda u)\|)}=-\frac{1}{V(\|q(\lambda u)\|)}\left\langle\lambda u-\frac{q(\lambda u)}{\|q(\lambda u)\|}, v_{k}\right\rangle \rightarrow \int_{\mathbb{R}^{d}} \frac{\left\langle y, v_{k}\right\rangle}{\|y-u\|} Q(y) d y
$$

as $\lambda \uparrow 1$. Besides, since $q(\lambda u) /\|q(\lambda u)\| \in S^{d-1}$, it is clear that

$$
b^{2}(\lambda)+\sum_{k=1}^{d-1} \beta_{k}^{2}(\lambda)=1 .
$$


In view of (3), $b(\lambda) \rightarrow 1$ as $\lambda \uparrow 1$ : it is thus easily seen from (42) that

$$
\begin{aligned}
\frac{1}{V(\|q(\lambda u)\|)}(b(\lambda)-1) & =-\frac{1}{2 V(\|q(\lambda u)\|)}\left(1-b^{2}(\lambda)\right)(1+\mathrm{o}(1)) \\
& =-V(\|q(\lambda u)\|)\left(\frac{1}{2} \sum_{k=1}^{d-1} \frac{\beta_{k}^{2}(\lambda)}{[V(\|q(\lambda u)\|)]^{2}}\right)(1+\mathrm{o}(1)) \rightarrow 0
\end{aligned}
$$

as $\lambda \uparrow 1$. Combining (40), (42), (44) and remarking that

$$
\Pi_{u}(y)=\sum_{k=1}^{d-1}\left\langle y, v_{k}\right\rangle v_{k}
$$

completes the first part of the proof.

(ii) Let us recall that, if $\alpha \in(1,2)$, then $\mathbb{E}\|X\|<\infty$. In this case, Lemma 1 in Girard and Stupfler (2015) shows that, for all $v \in \mathbb{R}^{d}$,

$$
\|q(\lambda u)\|\left\langle\lambda u-\frac{q(\lambda u)}{\|q(\lambda u)\|}, v\right\rangle \rightarrow-\mathbb{E}\left\langle\Pi_{u}(X), v\right\rangle \text { as } \lambda \uparrow 1 .
$$

Thus, Lemma 5 and (45) in the case $\alpha \leq 1$ and $\alpha \in(1,2)$ respectively show that for all $k \in\{1, \ldots, d-1\}$ :

$$
\frac{\beta_{k}^{2}(\lambda)}{V(\|q(\lambda u)\|)}=\left\{\begin{array}{ll}
\mathrm{O}(V(\|q(\lambda u)\|)) & \text { if } \alpha<1 \\
\mathrm{o}\left(\|q(\lambda u)\|^{1 / 2} V(\|q(\lambda u)\|)\right) & \text { if } \alpha=1 \\
\mathrm{O}\left(\left[\|q(\lambda u)\|^{2} V(\|q(\lambda u)\|)\right]^{-1}\right) & \text { if } 1<\alpha<2
\end{array}\right\}=\mathrm{o}(1)
$$

as $\lambda \uparrow 1$. Now, equation (43) and Lemma 6 yield

$$
\frac{1}{V(\|q(\lambda u)\|)}(\lambda b(\lambda)-1) \rightarrow-J(u) \text { as } \lambda \uparrow 1,
$$

and combining (43), (46) and (47) leads to

$$
\frac{1}{V(\|q(\lambda u)\|)}\left(1-\lambda b(\lambda)-\frac{1}{2}\left(1-b^{2}(\lambda)\right)\right) \rightarrow J(u) \text { as } \lambda \uparrow 1 .
$$

Finally, use once again either Lemma 5 if $\alpha \leq 1$ or equation (45) if $\alpha \in(1,2)$ to get

$$
\frac{1}{V(\|q(\lambda u)\|)}\left|\left\langle\lambda u-\frac{q(\lambda u)}{\|q(\lambda u)\|}, u\right\rangle\right|^{2}=\left\{\begin{array}{ll}
\mathrm{O}(V(\|q(\lambda u)\|)) & \text { if } \alpha<1 \\
\mathrm{o}\left(\|q(\lambda u)\|^{1 / 2} V(\|q(\lambda u)\|)\right) & \text { if } \alpha=1 \\
\mathrm{o}\left(\left[\|q(\lambda u)\|^{2} V(\|q(\lambda u)\|)\right]^{-1}\right) & \text { if } 1<\alpha<2
\end{array}\right\}=\mathrm{o}(1)
$$

as $\lambda \uparrow 1$ which implies

$$
\frac{1}{V(\|q(\lambda u)\|)}(\lambda-b(\lambda))^{2} \rightarrow 0 \text { as } \lambda \uparrow 1 .
$$

Using (48) together with (50) and the straightforward identity

$$
1-\lambda b(\lambda)-\frac{1}{2}\left(1-b^{2}(\lambda)\right)=\frac{1}{2}\left((1-\lambda)(1+\lambda)+(\lambda-b(\lambda))^{2}\right)
$$

yields the desired result. 


\subsection{Proof of Theorem 2}

Lemma 7 is the analogue of Lemma 5 when $\left(M_{1}\right)$ holds. It is the cornerstone to prove Theorem 2(i).

Lemma 7. Let $u \in S^{d-1}$. If $\left(M_{1}\right)$ holds with $\mathcal{L}(t) \rightarrow \infty$ as $t \rightarrow \infty$ then, for all $v \in \mathbb{R}^{d}$ and any symmetric positive definite $d \times d$ matrix $\Sigma$,

$$
\frac{\|q(\lambda u)\|}{\mathcal{L}(\|q(\lambda u)\|)}\left\langle\lambda u-\frac{q(\lambda u)}{\|q(\lambda u)\|}, v\right\rangle \rightarrow-\int_{E_{\Sigma}^{d-1}}\left\langle\Pi_{u}(w), v\right\rangle Q(w) \mu_{\Sigma}(d w) \text { as } \lambda \uparrow 1 .
$$

Proof of Lemma 7. The proof starts as the beginning of the proof of Lemma 5 (ii). Let $\mathcal{E}_{\Sigma}^{d}=\{x \in$ $\left.\mathbb{R}^{d} \mid x^{\prime} \Sigma^{-1} x \leq 1\right\}$. Since $\mathcal{E}_{\Sigma}^{d}$ is a compact neighborhood of $0,(24)$ entails, for $n$ large enough,

$$
\forall x \in \mathcal{E}_{\Sigma}^{d},\left|\frac{\langle x-q(\lambda u), v\rangle}{\|x-q(\lambda u)\|}+\left\langle\frac{q(\lambda u)}{\|q(\lambda u)\|}, v\right\rangle\right| \leq 2 \frac{\|v\|\|x\|}{\|q(\lambda u)\|}
$$

and therefore

$$
\int_{\mathcal{E}_{\Sigma}^{d}}\left(\frac{\langle x-q(\lambda u), v\rangle}{\|x-q(\lambda u)\|}+\left\langle\frac{q(\lambda u)}{\|q(\lambda u)\|}, v\right\rangle\right) f(x) d x=\mathrm{O}\left(\|q(\lambda u)\|^{-1}\right)
$$

where the boundedness of the function $y \mapsto\|y\|$ on the compact subset $\mathcal{E}_{\Sigma}^{d}$ of $\mathbb{R}^{d}$ was used. It thus follows from (2) that

$$
\int_{\mathbb{R}^{d} \backslash \mathcal{E}_{\Sigma}^{d}}\left(\frac{\langle x-q(\lambda u), v\rangle}{\|x-q(\lambda u)\|}+\left\langle\frac{q(\lambda u)}{\|q(\lambda u)\|}, v\right\rangle\right) f(x) d x+\left\langle\lambda u-\frac{q(\lambda u)}{\|q(\lambda u)\|}, v\right\rangle=\mathrm{O}\left(\|q(\lambda u)\|^{-1}\right) .
$$

Let us consider the Karamata representation (see Theorem 1.3.1 p.12 in Bingham et al., 1987) of the slowly varying function $\mathcal{L}$ defined in (4):

$$
\mathcal{L}(t)=c(t) \exp \left(\int_{1}^{t} \frac{\Delta(z)}{z} d z\right)
$$

where $c$ is a positive Borel measurable function converging to some positive constant, and $\Delta$ is a Borel measurable auxiliary function which converges to 0 at infinity. Lemma 1 then shows that there exists a function $\varepsilon_{1}:(1, \infty) \rightarrow[0, \infty)$ such that $\varepsilon_{1}(r) \rightarrow 0, r \varepsilon_{1}(r) \rightarrow \infty$,

$$
\varepsilon_{1}(r) \frac{\mathcal{L}\left(r \varepsilon_{1}(r)\right)}{L\left(r \varepsilon_{1}(r)\right)} \rightarrow \infty \text { and }\left|\log \varepsilon_{1}(r)\right| \sup _{z \geq r \varepsilon_{1}(r)}|\Delta(z)| \rightarrow 0 \text { as } r \rightarrow \infty .
$$

Let $\eta_{1}(\lambda)=\varepsilon_{1}(\|q(\lambda u)\|)$ for the sake of simplicity. Write further $f(y)=\|y\|^{-d} V(\|y\|) Q(y /\|y\|)(1+\theta(y))$ where $\theta(y) \rightarrow 0$ as $\|y\| \rightarrow \infty$ by Lemma 3 . Denote by $C_{1,-}$ (resp. $C_{1,+}$ ) the positive and finite infimum (resp. supremum) of the positive and continuous map $y \mapsto\|y\|$ on the compact subset $E_{\Sigma}^{d-1}$ of $\mathbb{R}^{d}$. Since $V$ can be taken continuous on $\left(C_{1,-} / 2, \infty\right)$, we may assume that $\theta$ is bounded on any annulus $\left\{y \in \mathbb{R}^{d} \mid C_{1,-} \leq\|y\| \leq C\right\}, C>C_{1,-}$. In view of $\mathcal{L}\left(\eta_{1}(\lambda)\|q(\lambda u)\|\right) \rightarrow \infty$ and (5), one has the expansion:

$$
\frac{\|q(\lambda u)\|}{\mathcal{L}\left(\eta_{1}(\lambda)\|q(\lambda u)\|\right)}\left\langle\lambda u-\frac{q(\lambda u)}{\|q(\lambda u)\|}, v\right\rangle=-\frac{\|q(\lambda u)\|}{\mathcal{L}\left(\eta_{1}(\lambda)\|q(\lambda u)\|\right)}\left(I_{1}(\lambda)+I_{2}(\lambda)\right)+\mathrm{o}(1)
$$

with

$$
\begin{aligned}
& I_{1}(\lambda)=\int_{1}^{\eta_{1}(\lambda)\|q(\lambda u)\|} \int_{E_{\Sigma}^{d-1}}\left(\frac{\langle r w-q(\lambda u), v\rangle}{\|r w-q(\lambda u)\|}+\left\langle\frac{q(\lambda u)}{\|q(\lambda u)\|}, v\right\rangle\right) r^{-2} L(r) Q(w)(1+\theta(r w)) d r \mu_{\Sigma}(d w), \\
& I_{2}(\lambda)=\int_{\eta_{1}(\lambda)\|q(\lambda u)\|}^{\infty} \int_{E_{\Sigma}^{d-1}}\left(\frac{\langle r w-q(\lambda u), v\rangle}{\|r w-q(\lambda u)\|}+\left\langle\frac{q(\lambda u)}{\|q(\lambda u)\|}, v\right\rangle\right) r^{-2} L(r) Q(w)(1+\theta(r w)) d r \mu_{\Sigma}(d w) .
\end{aligned}
$$


Let us start by controlling $I_{1}(\lambda)$. To this end, a Taylor expansion yields

$$
\sup _{w \in E_{\Sigma}^{d-1}} \sup _{z \in S^{d-1}}\left|\frac{1}{\rho}\left(\frac{\langle\rho w-z, v\rangle}{\|\rho w-z\|}+\langle z, v\rangle\right)-\left\langle\Pi_{z}(w), v\right\rangle\right| \rightarrow 0 \text { as } \rho \rightarrow 0,
$$

and therefore, in view of (3), we obtain

$$
\sup _{1 \leq r \leq \eta_{1}(\lambda)\|q(\lambda u)\|} \sup _{w \in E_{\Sigma}^{d-1}}\left|\frac{\|q(\lambda u)\|}{r}\left(\frac{\langle r w-q(\lambda u), v\rangle}{\|r w-q(\lambda u)\|}+\left\langle\frac{q(\lambda u)}{\|q(\lambda u)\|}, v\right\rangle\right)-\left\langle\Pi_{u}(w), v\right\rangle\right| \rightarrow 0 \text { as } \lambda \uparrow 1
$$

leading to

$$
\begin{aligned}
I_{1}(\lambda) & =\frac{1}{\|q(\lambda u)\|} \int_{1}^{\eta_{1}(\lambda)\|q(\lambda u)\|} \int_{E_{\Sigma}^{d-1}}\left\langle\Pi_{u}(w), v\right\rangle r^{-1} L(r) Q(w)(1+\theta(r w)) d r \mu_{\Sigma}(d w) \\
& +o\left(\frac{1}{\|q(\lambda u)\|} \int_{1}^{\eta_{1}(\lambda)\|q(\lambda u)\|} \int_{E_{\Sigma}^{d-1}}\left\langle\Pi_{u}(w), v\right\rangle r^{-1} L(r) Q(w)(1+|\theta(r w)|) d r \mu_{\Sigma}(d w)\right) .
\end{aligned}
$$

Besides,

$$
\frac{1}{\mathcal{L}\left(\eta_{1}(\lambda)\|q(\lambda u)\|\right)} \sup _{w \in E_{\Sigma}^{d-1}}\left(\int_{1}^{\eta_{1}(\lambda)\|q(\lambda u)\|} \frac{L(r)}{r}|\theta(r w)| d r\right) \rightarrow 0
$$

as $\lambda \uparrow 1$. Indeed, for any $C_{2}>1$, by separating the cases $r \in\left[1, C_{2}\right)$ and $r \geq C_{2}$, it appears that the left-hand side is less than

$$
\frac{\mathcal{L}\left(C_{2}\right)}{\mathcal{L}\left(\eta_{1}(\lambda)\|q(\lambda u)\|\right)} \sup _{C_{1,-} \leq\|y\| \leq C_{1,+} C_{2}}|\theta(y)|+\sup _{\|y\|>C_{1,+} C_{2}}|\theta(y)|
$$

which can be made arbitrarily small as $\lambda \uparrow 1$ by a suitable choice of the constant $C_{2}>1$. Hence the equality

$$
I_{1}(\lambda)=\frac{\mathcal{L}\left(\eta_{1}(\lambda)\|q(\lambda u)\|\right)}{\|q(\lambda u)\|}\left(\int_{E_{\Sigma}^{d-1}}\left\langle\Pi_{u}(w), v\right\rangle Q(w) \mu_{\Sigma}(d w)+\mathrm{o}(1)\right) .
$$

Let us turn to the term $I_{2}(\lambda)$, which, recalling $(26)$, can be bounded as follows:

$$
\left|I_{2}(\lambda)\right| \leq 2\|v\| \int_{\eta_{1}(\lambda)\|q(\lambda u)\|}^{\infty} r^{-2} L(r) d r \int_{E_{\Sigma}^{d-1}} Q(w) \mu_{\Sigma}(d w) .
$$

The change of variables $r=\eta_{1}(\lambda)\|q(\lambda u)\| \rho$ yields

$$
\left|I_{2}(\lambda)\right| \leq 2\|v\|\left(\eta_{1}(\lambda)\|q(\lambda u)\|\right)^{-1} L\left(\eta_{1}(\lambda)\|q(\lambda u)\|\right) \int_{1}^{\infty} \rho^{-2} \frac{L\left(\eta_{1}(\lambda)\|q(\lambda u)\| \rho\right)}{L\left(\eta_{1}(\lambda)\|q(\lambda u)\|\right)} d \rho \int_{E_{\Sigma}^{d-1}} Q(w) \mu_{\Sigma}(d w) .
$$

Let us introduce the function $h_{1}$ defined on $(0, \infty)$ by $h_{1}(\rho)=\rho^{-1 / 2} L(\rho)$. Clearly, $h_{1}$ is regularly varying with index $-1 / 2$ and therefore by a uniform convergence result (see e.g. Theorem 1.5.2 p.22 in Bingham et al., 1987):

$$
\sup _{\rho \geq 1}\left|\frac{h_{1}\left(\eta_{1}(\lambda)\|q(\lambda u)\| \rho\right)}{h_{1}\left(\eta_{1}(\lambda)\|q(\lambda u)\|\right)}-\rho^{-1 / 2}\right| \rightarrow 0 \quad \text { as } \quad \lambda \uparrow 1 .
$$

Since the function $\rho \mapsto \rho^{-3 / 2}$ is integrable over $[1, \infty)$, it follows that

$$
\left|I_{2}(\lambda)\right|=\mathrm{O}\left(\left(\eta_{1}(\lambda)\|q(\lambda u)\|\right)^{-1} L\left(\eta_{1}(\lambda)\|q(\lambda u)\|\right)\right)=\mathrm{o}\left(\|q(\lambda u)\|^{-1} \mathcal{L}\left(\eta_{1}(\lambda)\|q(\lambda u)\|\right)\right),
$$

in view of the properties of $\eta_{1}(\lambda)$. Combining (52), (54) and (55) then implies that

$$
\frac{\|q(\lambda u)\|}{\mathcal{L}\left(\eta_{1}(\lambda)\|q(\lambda u)\|\right)}\left\langle\lambda u-\frac{q(\lambda u)}{\|q(\lambda u)\|}, v\right\rangle \rightarrow-\int_{E_{\Sigma}^{d-1}}\left\langle\Pi_{u}(w), v\right\rangle Q(w) \mu_{\Sigma}(d w) \text { as } \lambda \uparrow 1 .
$$


Finally,

$$
\left|\int_{\eta_{1}(\lambda)\|q(\lambda u)\|}^{\|q(\lambda u)\|} \frac{\Delta(z)}{z} d z\right| \leq\left|\log \eta_{1}(\lambda)\right| \sup _{z \geq \eta_{1}(\lambda)\|q(\lambda u)\|}|\Delta(z)| \rightarrow 0 \quad \text { as } \quad \lambda \uparrow 1
$$

and therefore

$$
\frac{\mathcal{L}(\|q(\lambda u)\|)}{\mathcal{L}\left(\eta_{1}(\lambda)\|q(\lambda u)\|\right)}=\frac{c(\|q(\lambda u)\|)}{c\left(\eta_{1}(\lambda)\|q(\lambda u)\|\right)} \exp \left(\int_{\eta_{1}(\lambda)\|q(\lambda u)\|}^{\|q(\lambda u)\|} \frac{\Delta(z)}{z} d z\right) \rightarrow 1 \quad \text { as } \lambda \uparrow 1
$$

which completes the proof.

Lemma 8 is the analogue of Lemma 6 when $\left(M_{2}\right)$ holds. It will reveal useful to prove Theorem 2(ii).

Lemma 8. Let $u \in S^{d-1}$. If $\left(M_{2}\right)$ holds with $\mathcal{L}(t) \rightarrow \infty$ as $t \rightarrow \infty$ then, for any symmetric positive definite $d \times d$ matrix $\Sigma$,

$$
\frac{\|q(\lambda u)\|^{2}}{\mathcal{L}(\|q(\lambda u)\|)}\left\langle\lambda u-\frac{q(\lambda u)}{\|q(\lambda u)\|}, \frac{q(\lambda u)}{\|q(\lambda u)\|}\right\rangle \rightarrow-\frac{1}{2} \int_{E_{\Sigma}^{d-1}}\left\langle\Pi_{u}(w), w\right\rangle Q(w) \mu_{\Sigma}(d w) \text { as } \lambda \uparrow 1 .
$$

Proof of Lemma 8. Let us start as in the proof of Lemma 7, whose notation we retain. From (34), one has

$$
\left|1+\left\langle\frac{x-q(\lambda u)}{\|x-q(\lambda u)\|}, \frac{q(\lambda u)}{\|q(\lambda u)\|}\right\rangle\right| \leq \frac{\|x\|^{2}}{\|q(\lambda u)\|^{2}},
$$

for $n$ large enough and uniformly in $x \in \mathcal{E}_{\Sigma}^{d}$, leading to

$$
\int_{\mathcal{E}_{\Sigma}^{d}}\left(1+\left\langle\frac{x-q(\lambda u)}{\|x-q(\lambda u)\|}, \frac{q(\lambda u)}{\|q(\lambda u)\|}\right\rangle\right) f(x) d x=\mathrm{O}\left(\|q(\lambda u)\|^{-2}\right) .
$$

In view of $(2)$, it follows that

$$
\int_{\mathbb{R}^{d} \backslash \mathcal{E}_{\Sigma}^{d}}\left(1+\left\langle\frac{x-q(\lambda u)}{\|x-q(\lambda u)\|}, \frac{q(\lambda u)}{\|q(\lambda u)\|}\right\rangle\right) f(x) d x+\left\langle\lambda u-\frac{q(\lambda u)}{\|q(\lambda u)\|}, \frac{q(\lambda u)}{\|q(\lambda u)\|}\right\rangle=\mathrm{O}\left(\|q(\lambda u)\|^{-2}\right) .
$$

Besides, Lemma 1 shows that there exists a function $\varepsilon_{2}:(1, \infty) \rightarrow[0, \infty)$ such that $\varepsilon_{2}(r) \rightarrow 0, r \varepsilon_{2}(r) \rightarrow \infty$,

$$
\varepsilon_{2}^{2}(r) \frac{\mathcal{L}\left(r \varepsilon_{2}(r)\right)}{L\left(r \varepsilon_{2}(r)\right)} \rightarrow \infty \text { and }\left|\log \varepsilon_{2}(r)\right| \sup _{z \geq r \varepsilon_{2}(r)}|\Delta(z)| \rightarrow 0 \text { as } r \rightarrow \infty .
$$

Let us introduce $\eta_{2}(\lambda)=\varepsilon_{2}(\|q(\lambda u)\|)$. From (5), and since $\mathcal{L}\left(\eta_{2}(\lambda)\|q(\lambda u)\|\right) \rightarrow \infty$, it follows that

$$
\frac{\|q(\lambda u)\|^{2}}{\mathcal{L}\left(\eta_{2}(\lambda)\|q(\lambda u)\|\right)}\left\langle\lambda u-\frac{q(\lambda u)}{\|q(\lambda u)\|}, \frac{q(\lambda u)}{\|q(\lambda u)\|}\right\rangle=-\frac{\|q(\lambda u)\|^{2}}{\mathcal{L}\left(\eta_{2}(\lambda)\|q(\lambda u)\|\right)}\left[J_{1}(\lambda)+J_{2}(\lambda)\right]+\mathrm{o}(1)
$$

with

$$
\begin{aligned}
& J_{1}(\lambda)=\int_{1}^{\eta_{2}(\lambda)\|q(\lambda u)\|} \int_{E_{\Sigma}^{d-1}}\left(1+\left\langle\frac{r w-q(\lambda u)}{\|r w-q(\lambda u)\|}, \frac{q(\lambda u)}{\|q(\lambda u)\|}\right\rangle\right) r^{-3} L(r) Q(w)(1+\theta(r w)) d r \mu_{\Sigma}(d w), \\
& J_{2}(\lambda)=\int_{\eta_{2}(\lambda)\|q(\lambda u)\|}^{\infty} \int_{E_{\Sigma}^{d-1}}\left(1+\left\langle\frac{r w-q(\lambda u)}{\|r w-q(\lambda u)\|}, \frac{q(\lambda u)}{\|q(\lambda u)\|}\right\rangle\right) r^{-3} L(r) Q(w)(1+\theta(r w)) d r \mu_{\Sigma}(d w) .
\end{aligned}
$$

Let us start by controlling $J_{1}(\lambda)$. A Taylor expansion leads to

$$
\sup _{w \in E_{\Sigma}^{d-1}} \sup _{z \in S^{d-1}}\left|\frac{1}{\rho^{2}}\left(1+\frac{\langle\rho w-z, z\rangle}{\|\rho w-z\|}\right)-\frac{1}{2}\left\langle\Pi_{z}(w), w\right\rangle\right| \rightarrow 0 \text { as } \rho \rightarrow 0,
$$


and thus, in view of (3):

$$
\sup _{1 \leq r \leq \eta_{2}(\lambda)\|q(\lambda u)\|} \sup _{w \in E_{\Sigma}^{d-1}}\left|\frac{\|q(\lambda u)\|^{2}}{r^{2}}\left(1+\left\langle\frac{r w-q(\lambda u)}{\|r w-q(\lambda u)\|}, \frac{q(\lambda u)}{\|q(\lambda u)\|}\right\rangle\right)-\frac{1}{2}\left\langle\Pi_{u}(w), w\right\rangle\right| \rightarrow 0
$$

as $\lambda \uparrow 1$. This entails

$$
\begin{aligned}
J_{1}(\lambda) & =\frac{1}{\|q(\lambda u)\|^{2}} \int_{1}^{\eta_{2}(\lambda)\|q(\lambda u)\|} \int_{E_{\Sigma}^{d-1}} \frac{1}{2}\left\langle\Pi_{u}(w), w\right\rangle r^{-1} L(r) Q(w)(1+\theta(r w)) d r \mu_{\Sigma}(d w) \\
& +\mathrm{o}\left(\frac{1}{\|q(\lambda u)\|^{2}} \int_{1}^{\eta_{2}(\lambda)\|q(\lambda u)\|} \int_{E_{\Sigma}^{d-1}}\left\langle\Pi_{u}(w), w\right\rangle r^{-1} L(r) Q(w)(1+|\theta(r w)|) d r \mu_{\Sigma}(d w)\right) .
\end{aligned}
$$

Using (53) yields, as a preliminary conclusion:

$$
J_{1}(\lambda)=\frac{\mathcal{L}\left(\eta_{2}(\lambda)\|q(\lambda u)\|\right)}{\|q(\lambda u)\|^{2}}\left(\frac{1}{2} \int_{E_{\Sigma}^{d-1}}\left\langle\Pi_{u}(w), w\right\rangle Q(w) \mu_{\Sigma}(d w)+o(1)\right) .
$$

Let us turn to the term $J_{2}(\lambda)$, which can be bounded using the Cauchy-Schwarz inequality:

$$
\left|J_{2}(\lambda)\right| \leq 2 \int_{\eta_{2}(\lambda)\|q(\lambda u)\|}^{\infty} r^{-3} L(r) d r \int_{E_{\Sigma}^{d-1}} Q(w) \mu_{\Sigma}(d w) .
$$

The change of variables $r=\eta_{2}(\lambda)\|q(\lambda u)\| \rho$ yields

$$
\left|J_{2}(\lambda)\right| \leq 2\left(\eta_{2}(\lambda)\|q(\lambda u)\|\right)^{-2} L\left(\eta_{2}(\lambda)\|q(\lambda u)\|\right) \int_{1}^{\infty} \rho^{-3} \frac{L\left(\eta_{2}(\lambda)\|q(\lambda u)\| \rho\right)}{L\left(\eta_{2}(\lambda)\|q(\lambda u)\|\right)} d \rho \int_{E_{\Sigma}^{d-1}} Q(w) \mu_{\Sigma}(d w) .
$$

Let us consider the function $h_{2}$ defined on $(0, \infty)$ by $h_{2}(\rho)=\rho^{-1} L(\rho)$. Clearly, $h_{2}$ is regularly varying with index -1 and therefore by a uniform convergence result (see e.g. Theorem 1.5.2 p.22 in Bingham et al., 1987):

$$
\sup _{\rho \geq 1}\left|\frac{h_{2}\left(\eta_{2}(\lambda)\|q(\lambda u)\| \rho\right)}{h_{2}\left(\eta_{2}(\lambda)\|q(\lambda u)\|\right)}-\rho^{-1}\right| \rightarrow 0 \quad \text { as } \quad \lambda \uparrow 1 .
$$

Since the function $\rho \mapsto \rho^{-2}$ is integrable over $[1, \infty)$, it follows that

$$
\left|J_{2}(\lambda)\right|=\mathrm{O}\left(\left(\eta_{2}(\lambda)\|q(\lambda u)\|\right)^{-2} L\left(\eta_{2}(\lambda)\|q(\lambda u)\|\right)\right)=\mathrm{o}\left(\|q(\lambda u)\|^{-2} \mathcal{L}\left(\eta_{2}(\lambda)\|q(\lambda u)\|\right)\right)
$$

in view of the properties of $\eta_{2}(\lambda)$. Combining (56), (57) and (58) entails

$$
\frac{\|q(\lambda u)\|^{2}}{\mathcal{L}\left(\eta_{2}(\lambda)\|q(\lambda u)\|\right)}\left\langle\lambda u-\frac{q(\lambda u)}{\|q(\lambda u)\|}, \frac{q(\lambda u)}{\|q(\lambda u)\|}\right\rangle \rightarrow-\frac{1}{2} \int_{E_{\Sigma}^{d-1}}\left\langle\Pi_{u}(w), w\right\rangle Q(w) \mu_{\Sigma}(d w) .
$$

Finally, since

$$
\left|\int_{\eta_{2}(\lambda)\|q(\lambda u)\|}^{\|q(\lambda u)\|} \frac{\Delta(z)}{z} d z\right| \leq\left|\log \eta_{2}(\lambda)\right| \sup _{z \geq \eta_{2}(\lambda)\|q(\lambda u)\|}|\Delta(z)| \rightarrow 0 \text { as } \lambda \uparrow 1
$$

it is thus clear that

$$
\frac{\mathcal{L}(\|q(\lambda u)\|)}{\mathcal{L}\left(\eta_{2}(\lambda)\|q(\lambda u)\|\right)}=\frac{c(\|q(\lambda u)\|)}{c\left(\eta_{2}(\lambda)\|q(\lambda u)\|\right)} \exp \left(\int_{\eta_{2}(\lambda)\|q(\lambda u)\|}^{\|q(\lambda u)\|} \frac{\Delta(z)}{z} d z\right) \rightarrow 1 \text { as } \lambda \uparrow 1
$$

and the conclusion follows. 
Proof of Theorem 2. (i) We follow the lines of the proof of Theorem 1. Recalling the notation introduced in (40), Lemma 7 yields for all $k \in\{1, \ldots, d-1\}$ :

$$
\frac{\|q(\lambda u)\|}{\mathcal{L}(\|q(\lambda u)\|)} \beta_{k}(\lambda)=-\frac{\|q(\lambda u)\|}{\mathcal{L}(\|q(\lambda u)\|)}\left\langle\lambda u-\frac{q(\lambda u)}{\|q(\lambda u)\|}, v_{k}\right\rangle \rightarrow \int_{E_{\Sigma}^{d-1}}\left\langle w, v_{k}\right\rangle Q(w) \mu_{\Sigma}(d w)
$$

as $\lambda \uparrow 1$. The first statement is then proven by mimicking the proof of Theorem 1(i).

(ii) From Lemma 7, and in view of equations (41), (45), it follows that

$$
\beta_{k}^{2}(\lambda)=\mathrm{o}\left(\|q(\lambda u)\|^{-2} \mathcal{L}(\|q(\lambda u)\|)\right)
$$

as $\lambda \uparrow 1$ and for all $k \in\{1, \ldots, d-1\}$. Lemma 8 and equation (43) then yield

$$
\frac{\|q(\lambda u)\|^{2}}{\mathcal{L}(\|q(\lambda u)\|)}(\lambda b(\lambda)-1) \rightarrow-\frac{1}{2} \int_{E_{\Sigma}^{d-1}}\left\langle\Pi_{u}(w), w\right\rangle Q(w) \mu_{\Sigma}(d w)
$$

as $\lambda \uparrow 1$. Using once more equation (43), together with (59) and (60) entails

$$
\frac{\|q(\lambda u)\|^{2}}{\mathcal{L}(\|q(\lambda u)\|)}\left(1-\lambda b(\lambda)-\frac{1}{2}\left(1-b^{2}(\lambda)\right)\right) \rightarrow \frac{1}{2} \int_{E_{\Sigma}^{d-1}}\left\langle\Pi_{u}(w), w\right\rangle Q(w) \mu_{\Sigma}(d w)
$$

as $\lambda \uparrow 1$. One can thus use (61) to argue along the same lines as in the proof of Theorem 1(ii).

\subsection{Proof of Corollary 2}

Corollary 2 is a direct consequence of Theorem 2(ii) and of the following result:

Lemma 9. For all $u \in S^{d-1}$, one has

$$
\int_{E_{\Sigma}^{d-1}}\left\langle\Pi_{u}(w), w\right\rangle \mu_{\Sigma}(d w)=\frac{a_{d}}{d}(\operatorname{det} \Sigma)^{1 / 2}\left(\operatorname{tr} \Sigma-u^{\prime} \Sigma u\right)
$$

Proof of Lemma 9. The definition of $\mu_{\Sigma}$ entails

$$
\int_{E_{\Sigma}^{d-1}}\left\langle\Pi_{u}(w), w\right\rangle \mu_{\Sigma}(d w)=(\operatorname{det} \Sigma)^{1 / 2} \int_{S^{d-1}}\left\langle\Pi_{u}\left(\Sigma^{1 / 2} w\right), \Sigma^{1 / 2} w\right\rangle \sigma(d w) .
$$

Recalling that $\Pi_{u}(y)=y-\langle y, u\rangle u$ for any $y \in \mathbb{R}^{d}$, this yields

$$
\int_{E_{\Sigma}^{d-1}}\left\langle\Pi_{u}(w), w\right\rangle \mu_{\Sigma}(d w)=(\operatorname{det} \Sigma)^{1 / 2} \int_{S^{d-1}}\left(\left\|\Sigma^{1 / 2} w\right\|^{2}-\left\langle\Sigma^{1 / 2} w, u\right\rangle^{2}\right) \sigma(d w) .
$$

Let us denote by $m_{i j}$ the entry in the $i$-th row and $j$-th column of $\Sigma^{1 / 2}$. If $w=\left(w_{1}, \ldots, w_{d}\right)^{\prime}$ and $u=\left(u_{1}, \ldots, u_{d}\right)^{\prime}$, one has

$$
\left\|\Sigma^{1 / 2} w\right\|^{2}=\sum_{i, j=1}^{d} \sum_{k=1}^{d} m_{k i} m_{k j} w_{i} w_{j} \text { and }\left\langle\Sigma^{1 / 2} w, u\right\rangle^{2}=\sum_{i, j=1}^{d} \sum_{k, l=1}^{d} m_{k i} m_{l j} w_{i} w_{j} u_{k} u_{l} .
$$

Isotropy and symmetry arguments entail, for all $i, j \in\{1, \ldots, d\}$ with $i \neq j$ :

$$
\int_{S^{d-1}} w_{i}^{2} \sigma(d w)=\int_{S^{d-1}} w_{j}^{2} \sigma(d w) \text { and } \int_{S^{d-1}} w_{i} w_{j} \sigma(d w)=0 .
$$


Especially, since $w_{1}^{2}+\cdots+w_{d}^{2}=1$ for all $w \in S^{d-1}$, it follows that, for all $i \in\{1, \ldots, d\}$ :

$$
\int_{S^{d-1}} w_{i}^{2} \sigma(d w)=\frac{a_{d}}{d}
$$

Combining (62), (63) and (64) yields

$$
\int_{E_{\Sigma}^{d-1}}\left\langle\Pi_{u}(w), w\right\rangle \mu_{\Sigma}(d w)=\frac{a_{d}}{d}(\operatorname{det} \Sigma)^{1 / 2}\left(\sum_{i, k=1}^{d} m_{k i}^{2}-\sum_{i=1}^{d}\left[\sum_{k=1}^{d} m_{k i} u_{k}\right]^{2}\right) .
$$

Let us finally remark that, since $\Sigma^{1 / 2}$ is a symmetric matrix, it holds that

$$
\operatorname{tr} \Sigma=\sum_{i, k=1}^{d} m_{k i}^{2} \text { and } u^{\prime} \Sigma u=\sum_{i=1}^{d}\left(\sum_{k=1}^{d} m_{k i} u_{k}\right)^{2} .
$$

Plugging these two equalities into (65) concludes the proof.

\section{References}

Bingham, N.H., Goldie, C.M., Teugels, J.L. (1987) Regular Variation, Cambridge, U.K.: Cambridge University Press.

Breckling, J., Chambers, R. (1988) M-quantiles, Biometrika 75(4): 761-771.

Cadre, B., Gannoun, A. (2000) Asymptotic normality of consistent estimate of the conditional $L_{1}-$ median, Annales de l'Institut de Statistique de l'Université de Paris 44: 13-35.

Cai, J.-J., Einmahl, J.H.J., de Haan, L. (2011) Estimation of extreme risk regions under multivariate regular variation, The Annals of Statistics 39(3): 1803-1826.

Cambanis, S., Huang, S., Simons, G. (1981) On the theory of elliptically contoured distributions, Journal of Multivariate Analysis 11(3): 368-385.

Cardot, H., Cénac, P., Zitt, P.-A. (2013) Efficient and fast estimation of the geometric median in Hilbert spaces with an averaged stochastic gradient algorithm, Bernoulli 19(1): 18-43.

Chakraborty, B. (2001) On affine equivariant multivariate quantiles, Annals of the Institute of Statistical Mathematics 53(2): 380-403.

Chakraborty, B. (2003) On multivariate quantile regression, Journal of Statistical Planning and Inference 110(2): 109-132.

Chaouch, M., Goga, C. (2010) Design-based estimation for geometric quantiles with application to outlier detection, Computational Statistics and Data Analysis 54: 2214-2229.

Chaouch, M., Laïb, N. (2013) Nonparametric multivariate $L_{1}-$ median regression estimation with functional covariates, Electronic Journal of Statistics 7: 1553-1586. 
Chaudhuri, P. (1996) On a geometric notion of quantiles for multivariate data, Journal of the American Statistical Association 91(434): 862-872.

Cheng, Y., De Gooijer, J.G. (2007) On the $u$-th geometric conditional quantile, Journal of Statistical Planning and Inference 137: 1914-1930.

Chernozhukov, V. (2005) Extremal quantile regression, The Annals of Statistics 33(2): 806-839.

Daouia, A., Gardes, L., Girard, S. (2013) On kernel smoothing for extremal quantile regression, Bernoulli 19: $2557-2589$.

Dhar, S.S., Chakraborty, B., Chaudhuri, P. (2014) Comparison of multivariate distributions using quantilequantile plots and related tests, Bernoulli 20(3): 1484-1506.

Einmahl, J.H.J., de Haan, L., Krajina, A. (2013) Estimating extreme bivariate quantile regions, Extremes 16(2): $121-145$.

Gardes, L., Girard, S. (2012) Functional kernel estimators of large conditional quantiles, Electronic Journal of Statistics 6: 1715-1744.

Girard, S., Stupfler, G. (2015) Intriguing properties of extreme geometric quantiles, REVSTAT - Statistical Journal, to appear. Available at http://hal.inria.fr/hal-00865767.

de Haan, L., Ferreira, A. (2006) Extreme value theory: an introduction, Springer, New York.

Jessen, A.H., Mikosch, T. (2006) Regularly varying functions, Publications de l'Institut Mathématique (Beograd) 80(94): 171-192.

Kemperman, J.H.B. (1987) The median of a finite measure on a Banach space, in Statistical data analysis based on the $L^{1}$-norm and related methods, Ed. Y. Dodge, Amsterdam: North Holland, pp. $217-230$.

Koenker, R., Bassett, G. (1978) Regression quantiles, Econometrica 46: 33-50.

Koltchinskii, V.I. (2002) M-estimation, convexity and quantiles, Annals of Statistics 25(2): 435-477.

Resnick, S.I. (2006) Heavy tail phenomena: Probabilistic and statistical modeling, Springer Series in Operations Research and Financial Engineering, Springer-Verlag, New York.

Serfling, R. (2002) Quantile functions for multivariate analysis: approaches and applications, Statistica Neerlandica 56(2): 214-232.

Small, C.G. (1990) A survey of multidimensional medians, International Statistical Review 58(3): 263-277.

Stupfler, G. (2013) A moment estimator for the conditional extreme-value index, Electronic Journal of Statistics 7: 2298-2343. 
Weissman, I. (1978) Estimation of parameters and large quantiles based on the $k$ largest observations, Journal of the American Statistical Association 73: 812-815.

Acknowledgments. The authors thank two anonymous referees and the editor for their helpful suggestions and comments which contributed to an improved presentation of the results of this paper.

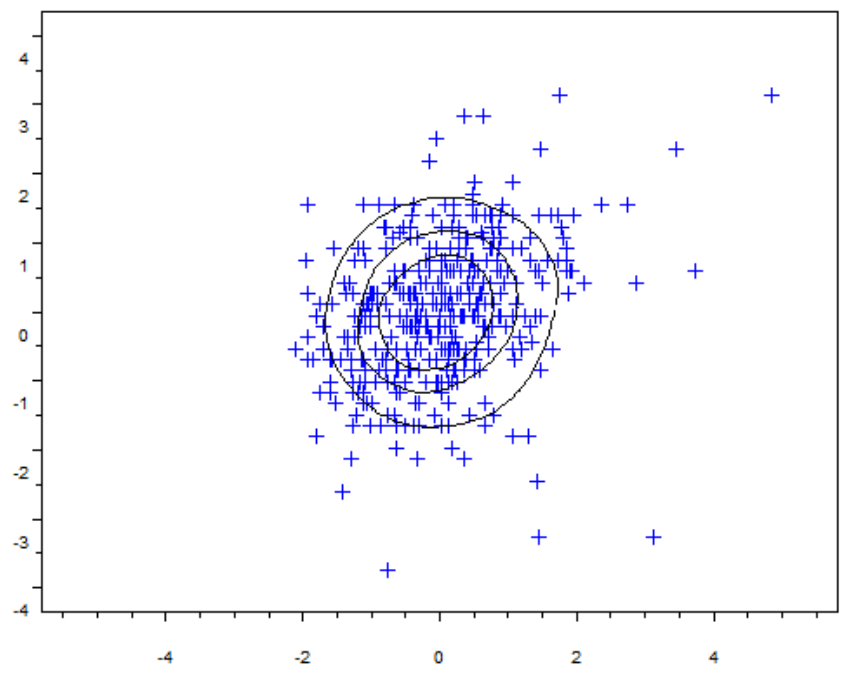

Figure 1: Three geometric iso-quantile curves $\{q(v),\|v\| \in\{0.5,0.65,0.8\}\}$ computed on a two-dimensional dataset extracted from the Pima Indians Diabetes Database. The data set consists of $n=392$ pairs $\left(X_{i}, Y_{i}\right)$, where $X_{i}$ is the body mass index of the $i$ th individual and $Y_{i}$ is its diastolic blood pressure. Both variables are centered and standardized. 

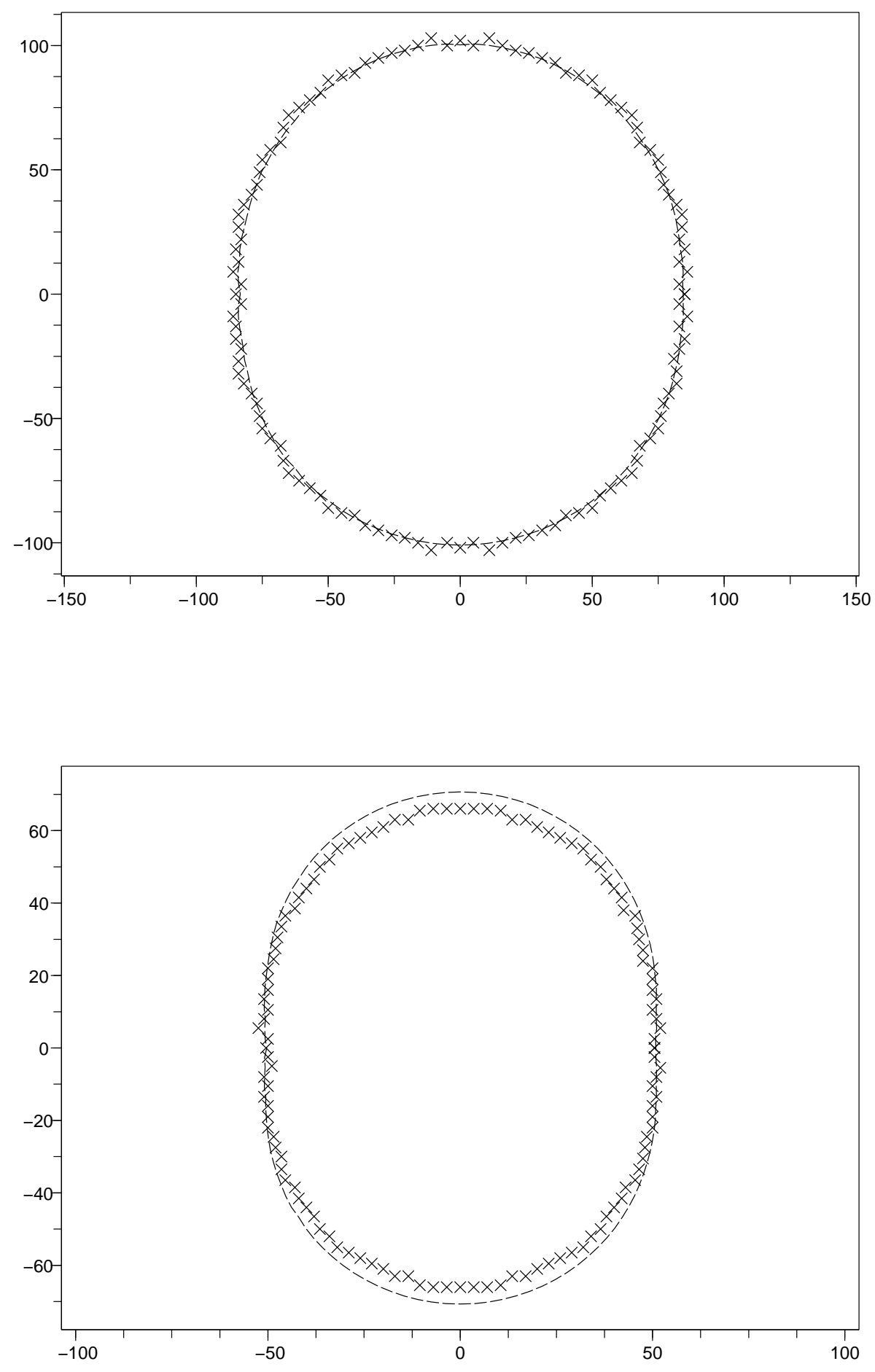

Figure 2: Extreme geometric quantiles from the Pareto model, top: $\alpha=1.3$, bottom: $\alpha=1.5$, dashed line: asymptotic equivalent, $\times$ : numerical computation. 

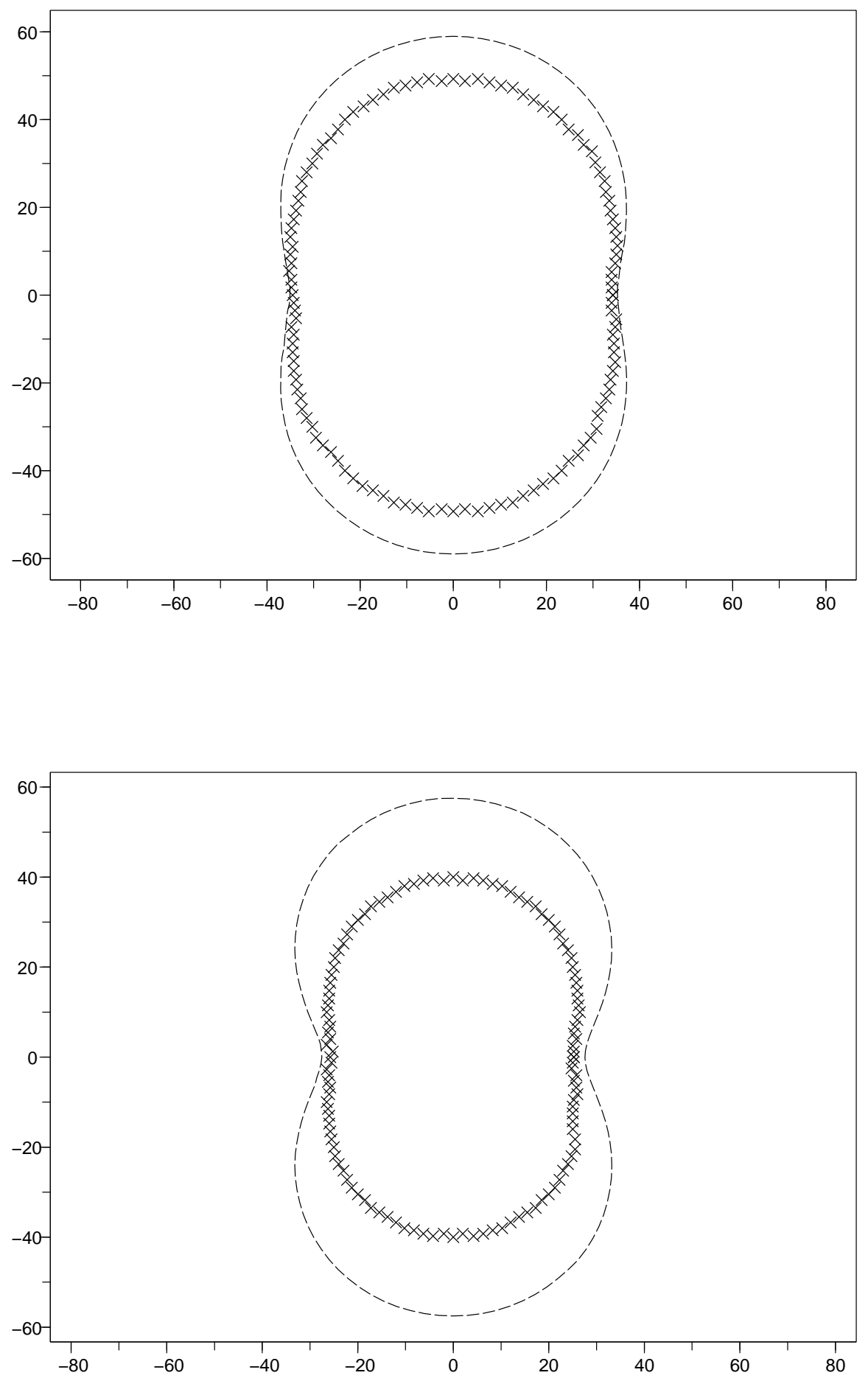

Figure 3: Extreme geometric quantiles from the Pareto model, top: $\alpha=1.7$, bottom: $\alpha=1.9$, dashed line: asymptotic equivalent, $\times$ : numerical computation. 

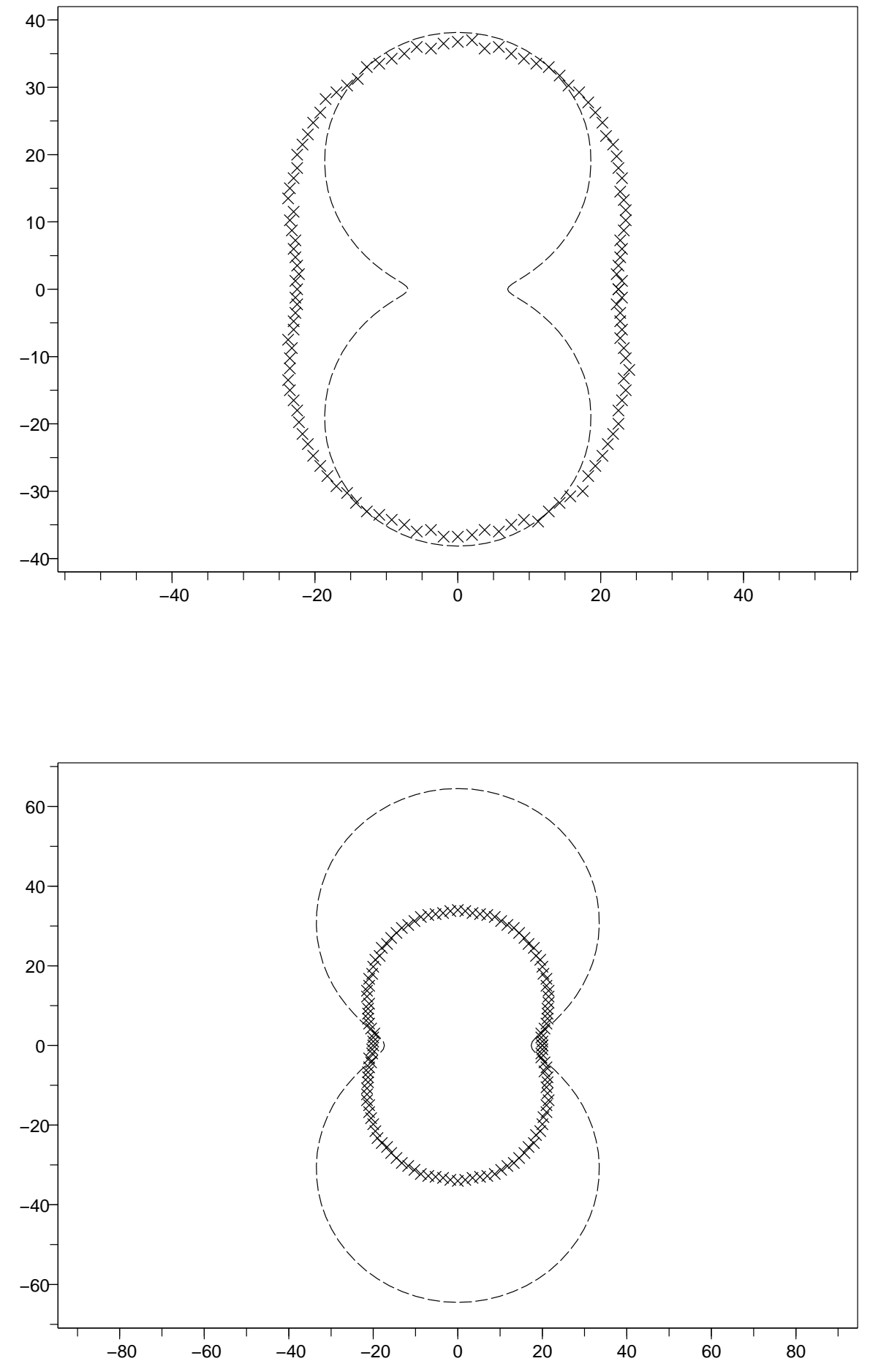

Figure 4: Extreme geometric quantiles from the Pareto model, top: $\alpha=2$, bottom: $\alpha=2.1$, dashed line: asymptotic equivalent, $\times$ : numerical computation. 

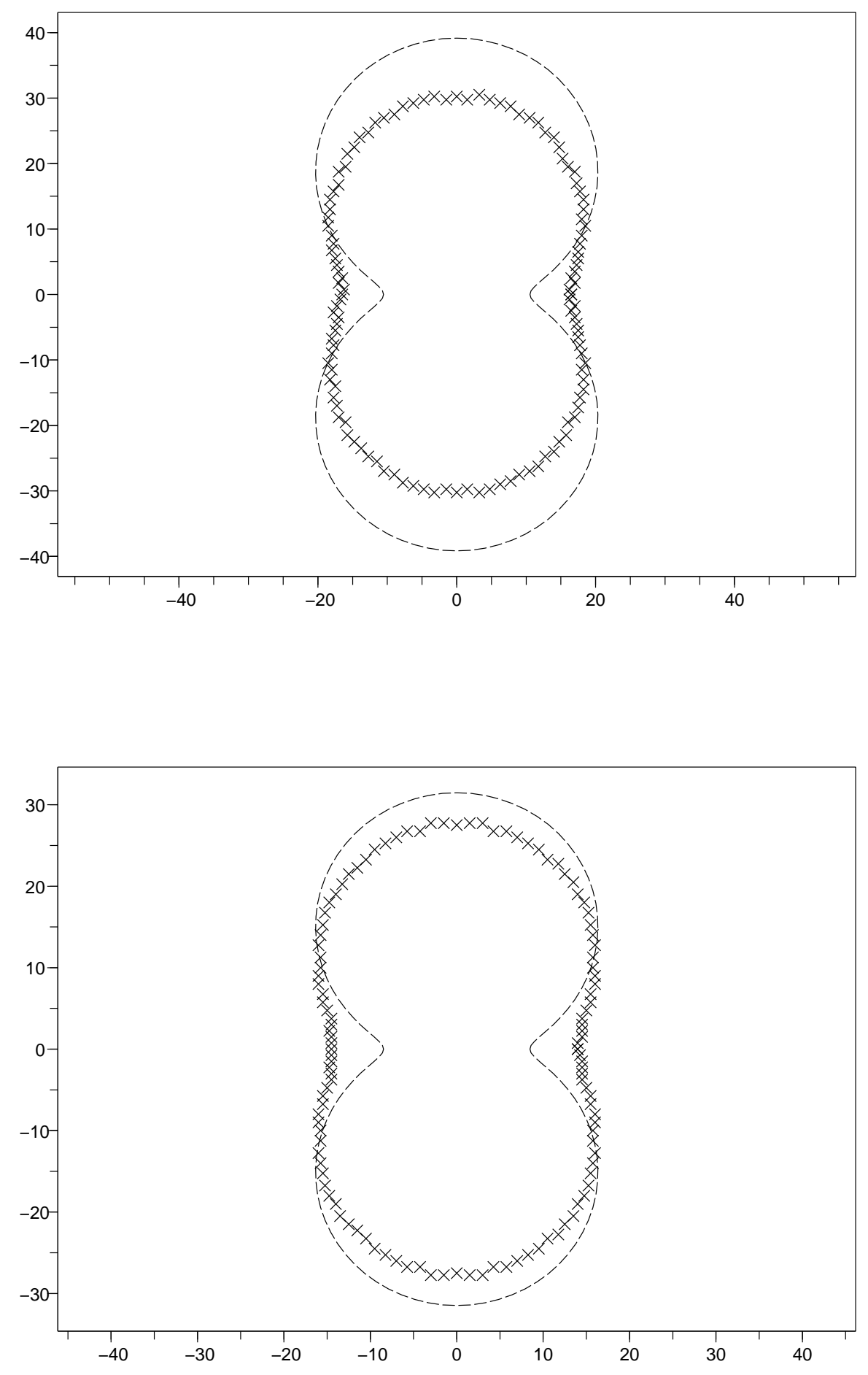

Figure 5: Extreme geometric quantiles from the Pareto model, top: $\alpha=2.3$, bottom: $\alpha=2.5$, dashed line: asymptotic equivalent, $\times$ : numerical computation. 

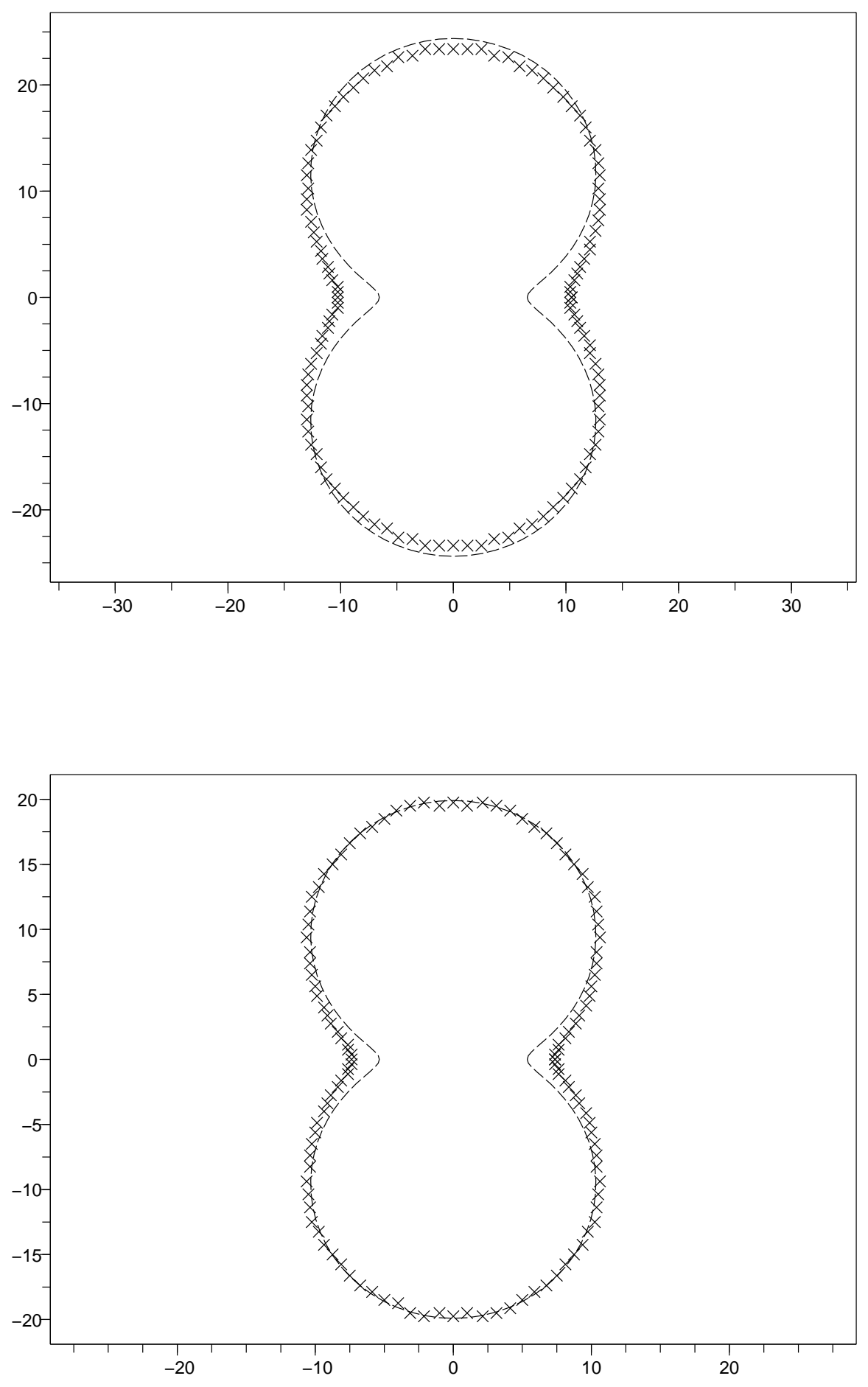

Figure 6: Extreme geometric quantiles from the Pareto model, top: $\alpha=3$, bottom: $\alpha=4$, dashed line: asymptotic equivalent, $\times$ : numerical computation. 